IMA Journal of Applied Mathematics (2005) 1-29

doi: 10.1093/imamat/

\title{
Quantum mechanical effects in continuum charge flow models
}

\author{
Jonathan P. Black, Christopher J. W. Breward*, Peter D. Howell $†$ \\ Mathematical Institute, University of Oxford, \\ Andrew Wiles Building, Oxford OX2 6GG, UK.
}

\begin{abstract}
This paper concerns mathematical modelling of charge transport across a thin poorly conducting layer between two electrodes. We describe and analyse two alternative approaches to model quantum effects within a continuum theory: the Density Gradient Confinement (DGC) and Density Gradient Tunnelling (DGT) theories. In either case, quantum effects are characterised by a small parameter, which we exploit to analyse the problems asymptotically. We thus find simplified approximate solutions which show excellent agreement with numerical solutions of the full models, and demonstrate previously undocumented oscillatory behaviour both in the charge density profile and in the variation of current with applied potential difference.
\end{abstract}

Keywords: Quantum drift-diffusion, asymptotic analysis, Density Gradient Tunnelling, Density Gradient Confinement

\section{Introduction}

Charge transport in semiconductor devices has been modelled using the drift-diffusion (Poisson-NernstPlanck) equations since the early 1950s (see Markowich (1986)). The modelling of semiconductor devices with the drift-diffusion equations increases understanding of device characteristics and enables optimal device design. However, the current drive towards minaturisation of electronic devices raises the question of whether or not quantum mechanical effects are important for simulating charge transport. Popular microscopic theories of charge transport, such as nonequilibrium Green's function methods (see Damle et al. (2001, 2002)), are very computationally expensive. The density gradient theories first formulated by Ancona and Tiersten (1987) provide a method to include quantum mechanical effects into the classical drift-diffusion equations, enabling efficient numerical solution and analysis using well developed analytical techniques. There are two different density gradient theories: (i) Density Gradient Confinement theory (DGC), and (ii) Density Gradient Tunnelling theory (DGT).

DGC theory was formulated by Ancona and Tiersten (1987) by assuming that the non-locality of quantum mechanics could be approximated by letting the energy densities of the electrons and holes depend on the spatial gradients of the charged particle densities. The DGC equations (often known as the quantum drift-diffusion equations) have since been derived in various ways; Cumberbatch et al. (2006) demonstrated that they can be obtained directly from the Schrödinger wave function for electrons expressed in amplitude/phase form, in contrast, Pinnau (2002) outlines the passage to DGC theory from

\footnotetext{
*Email: breward@maths.ox.ac.uk

${ }^{\dagger}$ Email: howell@maths.ox.ac.uk
} 
the kinetic Wigner-Poisson equation. The DGC governing equations take the form

$$
\begin{aligned}
\nabla^{2} \phi & =-\frac{q\left(n-p+N_{A}-N_{D}\right)}{\varepsilon}, \\
\frac{\partial n}{\partial t}+\nabla \cdot J_{n} & =G_{n}-U_{n}, \\
\frac{\partial p}{\partial t}+\nabla \cdot J_{p} & =G_{P}-U_{p}, \\
J_{n} & =-\frac{\mu_{n} k_{B} T}{q} \nabla n-\mu_{n} n \nabla \phi+\frac{\mu_{n} \hbar^{2}}{2 m_{n} r_{n}} n \nabla\left(\frac{\nabla^{2} \sqrt{n}}{\sqrt{n}}\right), \\
J_{p} & =-\frac{\mu_{p} k_{B} T}{q} \nabla p+\mu_{p} p \nabla \phi+\frac{\mu_{p} \hbar^{2}}{2 m_{p} r_{p}} p \nabla\left(\frac{\nabla^{2} \sqrt{p}}{\sqrt{p}}\right),
\end{aligned}
$$

where $\phi$ is the electric potential, $q$ is the charge on an electron, $n$ and $p$ are the electron and hole densities, respectively, $N_{A}$ and $N_{D}$ represent the concentration of acceptor impurities and donor impurities respectively, $\varepsilon$ is the absolute permittivity, $J_{n}$ and $J_{p}$ are the electron and hole fluxes, $G_{n}$ and $G_{P}$ are the generation rates of electrons and holes respectively, $U_{n}$ and $U_{p}$ are the corresponding recombination rates, $\mu_{n}$ and $\mu_{p}$ are the electron and hole mobilities, $k_{B}$ is Boltzmann's constant, $T$ is absolute temperature, $\hbar$ is Planck's constant, $m_{n}$ and $m_{p}$ are the electron and hole effective masses, respectively, and $r_{p}$ and $r_{n}$ are statistical factors (which are set to unity if the Pauli exclusion principle is ignored (see Ancona (2011))).

The DGC equations (1..1) differ from the drift-diffusion equations through the inclusion of the higher-order final terms in (1..1d) and (1..1e) that arise from the so-called quantum or Bohm potential. These terms provide additional non-local driving forces on the charged particles (see Ancona et al. (2002); Sano et al. (2002); Watling et al. (2002)), and their coefficients measure the strength of the gradient dependence of the transport of the charged particles (see Ancona (1990); Ancona et al. (2000)). It is often assumed that the non-local terms in (1..1d) and (1..1e) are small relative to drift and diffusion, making the equations suitable for singular perturbation analysis. However, singular perturbation analysis of the DGC equations has only been carried out in a select few studies (see Cumberbatch et al. (2006); Uno et al. (2007)).

The DGC equations are the lowest-order quantum correction and therefore are only strictly applicable when quantum effects can be considered as a small perturbation to a classical system (see Ancona and Iaafrate (1989)). However, with the values of the effective masses and statistical factors used as fitting parameters, the DGC equations have been shown to give good qualitative agreement with tunnelling experiments (see Ancona et al. (1997); Ancona (2011); Watling et al. (2002)) and therefore have been used in number of studies to model metal-oxide-semiconductor (MOS) devices where quantum mechanical effects are significant (see Ancona et al. (1997, 2002); Ancona (2011); Asenov et al. (2001); Cumberbatch et al. (2006); Abebe and Cumberbatch (2003); Uno et al. (2007)).

The alternative DGT theory was formulated to model charge transport on length scales of order $1 \mathrm{~nm}$, much smaller than the mean free path of an electron, so that scattering of charged particles is negligible and the conduction of electrons is governed by Newton's second law of motion (ballistic transport) (see Ancona (2011)). The governing equations are derived by making the following modifications to (1..1).

- Recombination and generation are neglected.

- As the scattering of charged particles is assumed to be negligible, the limit $\mu_{n}, \mu_{p} \rightarrow \infty$ is taken. The electron and hole densities are therefore known as the tunnelling electron density $n_{t}$ and 
tunnelling hole density $p_{t}$. In this limit the quasi-fermi level is constant and therefore the system is in quasi-thermal equilibrium.

- Inertial effects are included in (1..1d)-(1..1e).

The resulting equations are therefore

$$
\begin{aligned}
\nabla^{2} \phi & =-\frac{q\left(n_{t}-p_{t}+N_{A}-N_{D}\right)}{\varepsilon}, \\
\frac{\partial n_{t}}{\partial t}+\nabla \cdot J_{n} & =0 \\
\frac{\partial p_{t}}{\partial t}+\nabla \cdot J_{p} & =0 \\
n_{t} m_{n}\left(\frac{\partial v_{n}}{\partial t}+\left(v_{n} \cdot \nabla\right) v_{n}\right) & =-\frac{k_{B} T}{n_{t}} \nabla n_{t}-q n_{t} \nabla \phi+\frac{n_{t} \hbar^{2}}{2 m_{n} r_{n}} \nabla\left(\frac{\nabla^{2} \sqrt{n_{t}}}{\sqrt{n_{t}}}\right), \\
p_{t} m_{p}\left(\frac{\partial v_{p}}{\partial t}+\left(v_{p} \cdot \nabla\right) v_{p}\right) & =-\frac{k_{B} T}{p_{t}} \nabla p_{t}+q p_{t} \nabla \phi+\frac{p_{t} \hbar^{2}}{2 m_{p} r_{p}} \nabla\left(\frac{\nabla^{2} \sqrt{p_{t}}}{\sqrt{p_{t}}}\right),
\end{aligned}
$$

where $v_{n}, v_{p}$ are the electron and hole gas velocities, respectively, and $J_{n}, J_{p}$ are the electron and hole fluxes, respectively. Furthermore, since there is negligible interaction between the charge carriers, the electron/hole populations that are injected from different electrodes must have their own transport equations (see Ancona (1990); Ancona et al. (2000); Ancona \& Svizhenko (2008)). Making use of fitting parameters, good agreement with experiment has been found when using (1..2) to model tunnelling in ultrathin MOS capacitors (see Ancona et al. (2000)).

In $\S 2$., we use DGC theory to introduce quantum mechanical effects into a one-dimensional model of electron flow across a glass layer previously investigated in Black et al. (2013). We find that quantum mechanical effects alter the distribution of the electron density in the glass layer but are unmeasurable on the macroscopic scale. In $\S 3$, we investigate DGT theory by extending the work of Ancona (1990) on modelling charge transport in metal-insulator-metal devices. We use asymptotic and numerical analysis to characterise the solution structure of the electron density within the insulator. Finally, in $\S 4$., we summarise our findings and compare the two theories.

\section{Density Gradient Confinement theory}

\subsection{Formulation of the problem}

Our problem formulation takes the same form as given in Black et al. (2013); a schematic is given in Figure 1. We consider one-dimensional conduction through a glass layer of thickness $L$ across which a potential difference $\bar{\Phi}$ is imposed. We consider two systems: (i) glass separating a silicon electrode from a silver electode (Si-glass-Ag system); (ii) glass separating two silver electrodes (Ag-glass-Ag system). We denote the electron densities at the two electrodes $x=0$ and $x=L$ by $n_{0}$ and $n_{1}$ respectively; in the Ag-glass-Ag system we expect $n_{0} \approx n_{1}$ and in the Si-glass-Ag system we expect $n_{0}<n_{1}$. In practice, the values of $n_{0}$ and $n_{1}$ depend on the electron densities and affinities of the glass, silicon, and silver but, as in Black et al. (2013), here we take $n_{0}$ and $n_{1}$ to be known constants; the validity of this assumption is considered in Chapter 5 of Black (2015). 


$$
\begin{array}{r}
\text { Silicon/silver electri } \\
\phi=\Phi \\
n=n_{0}
\end{array}
$$

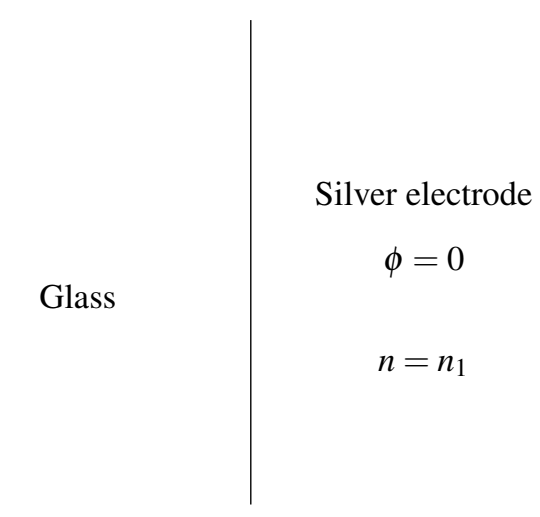

$x=L$

FIG. 1. Schematic of the problem modelled using DGC theory.

As in Black et al. (2013), we simplify the full DGC equations (1..1) by making the following assumptions.

1. The motion of holes is ignored and charge is therefore predominantly carried by electrons.

2. The system is in quasi-steady state.

3. The are no donor or acceptor impurities in the glass layer.

By following the same nondimensionalisation as in Black et al. (2013), we obtain the following normalised governing equations:

$$
\begin{aligned}
\frac{\mathrm{d} j}{\mathrm{~d} x} & =0, \\
j & =-n \frac{\mathrm{d} \phi}{\mathrm{d} x}-\frac{\mathrm{d} n}{\mathrm{~d} x}+\delta^{2} n \frac{\mathrm{d}}{\mathrm{d} x}\left(\frac{1}{\sqrt{n}} \frac{\mathrm{d}^{2} \sqrt{n}}{\mathrm{~d} x^{2}}\right), \\
v^{2} \frac{\mathrm{d}^{2} \phi}{\mathrm{d} x^{2}} & =-n,
\end{aligned}
$$

and the boundary conditions are

$$
\begin{aligned}
n(0) & =\alpha, & \phi(0) & =\Phi, \\
n(1) & =1, & \frac{\delta^{2}}{\sqrt{n}} \frac{\mathrm{d}^{2} \sqrt{n}}{\mathrm{~d} x^{2}}(0) & =0, \\
\phi(1) & =0, & \frac{\delta^{2}}{\sqrt{n}} \frac{\mathrm{d}^{2} \sqrt{n}}{\mathrm{~d} x^{2}}(1) & =0 .
\end{aligned}
$$

The quantum boundary conditions in (2..4) are formulated by making the assumption that quantum mechanical effects are not present in the electrodes and therefore the quantum potential is zero at the boundaries (see Pinnau (1999, 2002)).

The dimensionless parameters in the problem $(2 . .1)-(2 . .4)$ are

$$
\alpha=\frac{n_{0}}{n_{1}}, \quad \quad \quad \quad \quad=\frac{q \bar{\Phi}}{k_{B} T}, \quad \delta=\frac{L_{D}}{L}, \quad \delta=\frac{L_{Q}}{L},
$$




\begin{tabular}{ccccc} 
Parameter & Symbol & Value & Units & Notes \\
\hline Boltzmann's constant & $k_{B}$ & $1.38 \times 10^{-23}$ & $\mathrm{~m}^{2} \mathrm{~kg} \mathrm{~s}^{-2} \mathrm{~K}^{-1}$ & \\
Planck's constant & $\hbar$ & $1.04 \times 10^{-34}$ & $\mathrm{~m}^{2} \mathrm{~kg} \mathrm{~s}^{-1}$ & \\
Elementary charge & $q$ & $1.60 \times 10^{-19}$ & $\mathrm{C}$ & \\
Applied potential difference & $\bar{\Phi}$ & 0.6 & $\mathrm{~V}$ & \\
Glass layer thickness & $L$ & $10^{-8}-10^{-6}$ & $\mathrm{~m}$ & \\
Temperature & $T$ & 300 & $\mathrm{~K}$ & \\
Absolute permittivity & $\varepsilon$ & $3.45 \times 10^{-11}$ & $\mathrm{~F} \mathrm{~m}^{-1}$ & $(1)$ \\
Effective electron mass & $m_{n}$ & $9.11 \times 10^{-31}$ & $\mathrm{~kg}$ & $(2)$ \\
Statistical factor & $r_{n}$ & 3 & - & $(3)$ \\
Interfacial electron density & $n_{1}$ & $\sim 10^{25}$ & $\mathrm{~m}^{-3}$ & $(4)$
\end{tabular}

Table 1. Typical values of physical parameters for DGC model. (1) Here we use the absolute permittivity of silicon dioxide to estimate $\varepsilon$. (2) This value is the mass of a free electron, which is of the same order as the estimated effective electron mass in silicon (see Sze \& Ng (2006)) and the various estimated values for silicon dioxide (see Verwey et al. (1990)). (3) This is the value often found in "high" temperature experiments (see Ancona (1992)). (4) For comparison, the electron density in highly doped silicon is $\approx 10^{26} \mathrm{~m}^{-3}$ (see Sze \& $\mathrm{Ng}(2006)$ ).

where

$$
L_{D}=\sqrt{\frac{\varepsilon k_{B} T}{q^{2} n_{1}}} \quad \text { and } \quad L_{Q}=\frac{\hbar}{\sqrt{2 m_{n} r_{n} k_{B} T}}
$$

are two intrinsic length-scales known as the Debye length and the electron quantum length respectively. The Debye length gives the length-scale over which the system achieves approximate charge neutrality; the electron quantum length defines the length scale over which quantum mechanical effects are significant, and is of the order of the de Broglie wavelength associated with an electron (see Ancona (2011)). The parameter values given in Table 1 allow us to estimate

$$
L_{D} \approx 7.5 \times 10^{-10} \mathrm{~m}, \quad L_{Q} \approx 6.9 \times 10^{-10} \mathrm{~m} .
$$

Hence $L_{Q}$ and $L_{D}$ are similar in size and both much smaller than a typical glass layer thickness. This implies that $\delta$ and $v$ are both small parameters which are approximately of the same order. For the ratio $\alpha$ between the electron densities at the electrodes we expect $\alpha \approx 1$ in the Ag-glass-Ag system and $\alpha \lesssim 1$ in the Si-glass-Ag system. For the normalised applied potential $\Phi$, the values in Table 1 suggest $\Phi \approx 23$.

To solve the problem numerically, we eliminate $j$ from (2..1)-(2..4) to obtain the sixth-order system

$$
\frac{\mathrm{d}}{\mathrm{d} x}\left[\delta^{2} n \frac{\mathrm{d}}{\mathrm{d} x}\left(\frac{1}{\sqrt{n}} \frac{\mathrm{d}^{2} \sqrt{n}}{\mathrm{~d} x^{2}}\right)-n \frac{\mathrm{d} \phi}{\mathrm{d} x}-\frac{\mathrm{d} n}{\mathrm{~d} x}\right]=0, \quad \quad v^{2} \frac{\mathrm{d}^{2} \phi}{\mathrm{d} x^{2}}=-n .
$$

Given the values of $\Phi, \alpha, v$ and $\delta$, we solve (2..8) as a boundary-value problem for $n(x)$ and $\phi(x)$, subject to the boundary conditions (2..4). We then obtain the corresponding value of the flux $j$ using

$$
j=\frac{\Phi+\log \alpha}{\int_{0}^{1} \frac{\mathrm{d} x}{n(x)}}
$$

The evaluation of the integral in (2..9) and the numerical solution of (2..8) are performed using the spectral software package Chebfun. The numerical results are plotted below after we have performed the corresponding asymptotic analysis. 


\subsection{Asymptotic analysis}

We recall that the parameter $\delta$ is small, and the effects of the quantum term in equation (2..2) are therefore also small over the length-scale of the glass layer thickness. If we just set $\delta=0$, then the problem (2..1)-(2..4) reduces to the the drift-diffusion (DD) model proposed in (see Black et al. (2013)), and our aim is to determine how the solution is affected by inclusion of the quantum term proportional to $\delta^{2}$. Since $\delta$ multiplies the highest derivative in equation (2..2), the limit $\delta \rightarrow 0$ is singular. We therefore anticipate the existence of quantum boundary layers close to the edges $x=0,1$ of the domain, in which the full solution may depart significantly from the solution of the DD model. The structure of these layers depends on the sizes of the other parameters in the problem compared with $\delta$. We will assume henceforth that $\alpha=O(1)$. The typical parameter values given in Table 1, suggest that $v$ is roughly the same size as $\delta$, so we will let $v=O(\delta)$ as $\delta \rightarrow 0$. This scaling ensures that the quantum boundary layers have the same thickness as the Debye layers, in which there are significant space charge effects.

Finally, there are two scaling choices for the flux $j$ that result in distinguished limits. When $v$ is small, the glass layer is a poor conductor so that the current is also typically small, and a dominant balance in the Poisson equation (2..3) suggests the "small current" scaling $j=O\left(v^{2}\right)$. On the other hand, we recall that the dimensionless potential difference $\Phi$ is typically quite large, possibly large enough to drive a significant current despite the assumed smallness of $v$. The alternative "large current" scaling $j=O\left(v^{-2}\right)$ allows $O(1)$ variations in the electron density $n$ when $v$ is small.

Of course there are many other possible scalings for $v$ and $j$, for example $v=O(1)$ and $j=O(1)$ as $\delta \rightarrow 0$. However, all such alternative choices result either in simplified versions of the distinguished limits identified above or in solutions with weak boundary layers in which quantum effects are everywhere negligible. We therefore focus our efforts on the "small current" and "large current" regimes, which are analysed below in $\S 2.3$. and $\S 2.4$. respectively.

\subsection{Small current regime}

The limit $v \rightarrow 0$ for the DD problem was analysed in Black et al. (2013). The basic structure consists of an outer region, in which the electron density $n$ is $O\left(v^{2}\right)$, and Debye layers of thickness $O(v)$, in which $n$ adjusts rapidly to the imposed values $n=\alpha$ and $n=1$ at $x=0$ and $x=1$ respectively. Here we consider the regime in which $v=O(\delta)$ as $\delta \rightarrow 0$, so that the Debye layers and the quantum boundary layers coincide. The leading-order outer problem to be solved away from these layers is therefore identical to that studied in Black et al. (2013). The leading-order outer solutions for $n$ and $\phi$ take the forms

$$
n(x)=-2 v^{2} \frac{\mathrm{d}^{2}}{\mathrm{~d} x^{2}}(\log U(x)), \quad \phi(x)=2 \log \left(\frac{U(x)}{v}\right),
$$

where

$$
U(x)=a \operatorname{Ai}(c x-w)+b \operatorname{Bi}(c x-w), \quad c=\frac{j}{2 v^{2}},
$$

and $a, b, w$ are integration constants.

To satisfy the boundary conditions (2..4), we must introduce boundary layers at $x=0$ and $x=1$ in which $n$ is $O(1)$. First considering $x=0$, we make the scalings

$$
x=v \sqrt{\frac{2}{\alpha}} X, \quad n(x)=\alpha N(X), \quad \phi(x)=\Phi+\theta(X),
$$


before letting $v \sim \delta \rightarrow 0$, to obtain the leading-order system

$$
\frac{D}{2 \sqrt{N}} \frac{\mathrm{d}^{2} \sqrt{N}}{\mathrm{~d} X^{2}}=\theta+\log N, \quad \frac{1}{2} \frac{\mathrm{d}^{2} \theta}{\mathrm{d} X^{2}}=-N
$$

where

$$
D=\frac{\delta^{2} \alpha}{v^{2}}
$$

The boundary conditions (2..4a) lead to the conditions

$$
N=1, \quad \theta=0 \quad \text { at } X=0 .
$$

To match with the outer solution (2..10), we require $N=O\left(1 / X^{2}\right)$ as $X \rightarrow \infty$, and it follows that $\theta$ and $N$ must have the far-field behaviour

$$
\theta \sim 2 \log X, \quad N \sim \frac{1}{X^{2}} \quad \text { as } X \rightarrow \infty
$$

For the boundary layer at $x=1$, we follow an analogous procedure, but with $\Phi=0$ and $\alpha=1$, to obtain the same problem (2..13)-(2..16), but now with $D=\delta^{2} / v^{2}$. Therefore, to find the profiles for $n$ and $\phi$ in both boundary layers, it remains to solve the coupled equations (2..13), subject to the boundary and matching conditions (2..15) and (2..16).

We solve the problem (2..13)-(2..16) numerically by shooting from $X \rightarrow \infty$. The procedure is simplified by introducing rescaled variables $\tilde{X}, \tilde{N}$ and $\tilde{\theta}$ such that

$$
X=\sqrt{D}\left(\tilde{X}-\tilde{X}_{0}\right), \quad \quad N(X)=\frac{1}{D} \tilde{N}(\tilde{X}), \quad \theta(X)=\log D+\tilde{\theta}(\tilde{X}),
$$

resulting in the transformed problem

$$
\begin{array}{rlr}
\frac{1}{2 \sqrt{\tilde{N}}} \frac{\mathrm{d}^{2} \sqrt{\tilde{N}}}{\mathrm{~d} \tilde{X}^{2}}=\tilde{\theta}+\log \tilde{N}, & \frac{1}{2} \frac{\mathrm{d}^{2} \tilde{\theta}}{\mathrm{d} \tilde{X}^{2}}=-\tilde{N}, \\
\tilde{N}=D, \quad \tilde{\theta}=-\log D & \text { at } \tilde{X}=\tilde{X}_{0}, \\
\tilde{N} \sim \frac{1}{\tilde{X}^{2}}, \quad \tilde{\theta} \sim 2 \log \tilde{X} & \text { as } \tilde{X} \rightarrow \infty
\end{array}
$$

To facilitate the shooting, we consider the far-field conditions (2..20) more closely. We find that, up to an arbitrary translation, all possible solutions of (2..18) satisfying (2..20) take the asymptotic forms

$$
\begin{aligned}
& \tilde{\theta}(\tilde{X}) \sim 2 \log \tilde{X}-\frac{1}{2 \tilde{X}^{2}}-\frac{13}{4 \tilde{X}^{4}}+\cdots-\frac{C}{2 \tilde{X}} \mathrm{e}^{-2 \tilde{X}}, \\
& \tilde{N}(\tilde{X}) \sim \frac{1}{\tilde{X}^{2}}+\frac{3}{2 \tilde{X}^{4}}+\frac{65}{4 \tilde{X}^{6}}+\cdots \frac{C}{\tilde{X}} \mathrm{e}^{-2 \tilde{X}}
\end{aligned}
$$

as $\tilde{X} \rightarrow \infty$, where $C$ is an arbitrary constant.

For a chosen value of the shooting parameter $C$, we solve the system (2..18) subject to the far-field behaviour (2..21). We shoot until the condition

$$
\tilde{\theta}+\log \tilde{N}=0 \quad \text { at } \tilde{X}=\tilde{X}_{0}
$$



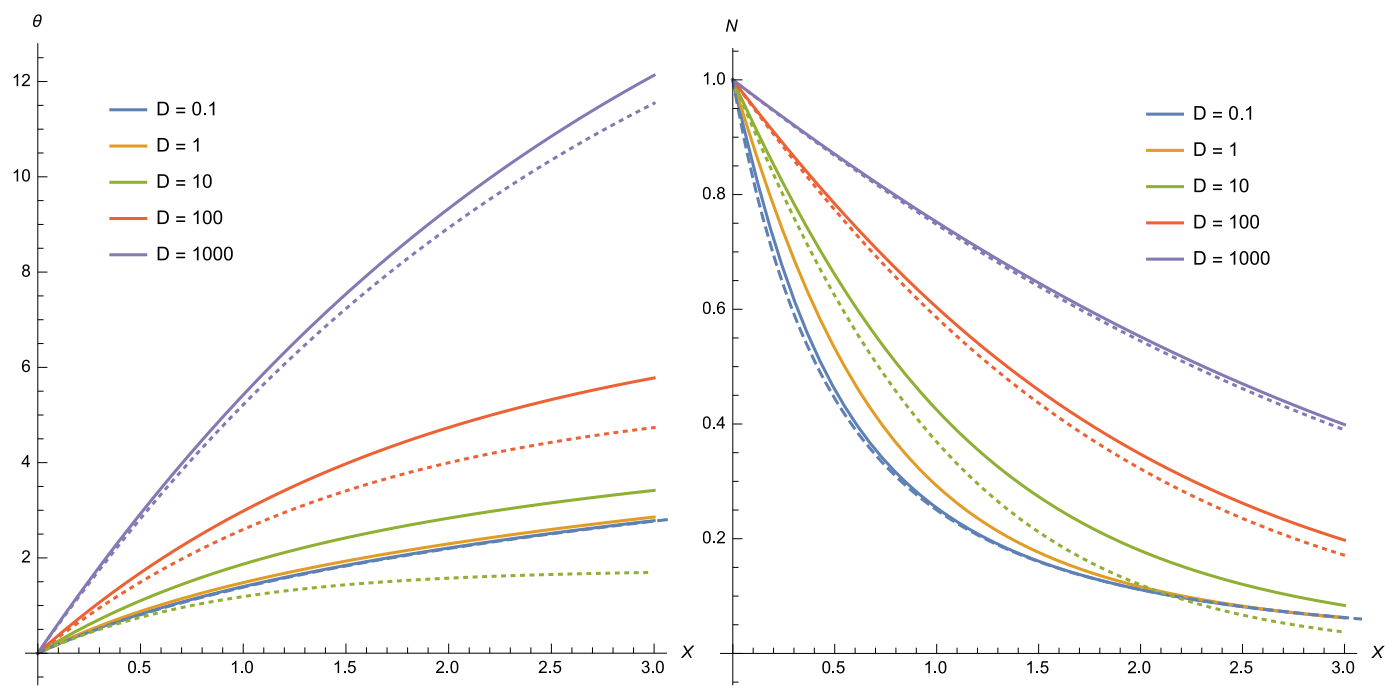

FIG. 2. Normalised electric potential $\theta$ and electron density $N$ plotted against scaled position $X$ in the boundary layer, for different values of the scaled quantum parameter $D$. The solid curves are the numerical solution obtained using the shooting method. The blue dashed curve shows the small- $D$ limit (2..23). The dotted curves show the large- $D$ approximation (2..29) for $D=10,100,1000$.

is satisfied, and then read off the corresponding value of $D=\tilde{N}\left(\tilde{X}_{0}\right)$. A specified value of $D$ is thus achieved by varying the shooting parameter $C$, and the solution in the original variables is then obtained by reversing the scalings $(2 . .17)$.

Numerical solutions for $\theta(X)$ and $N(X)$ obtained by following this procedure are plotted as solid curves in Figure 2 for various values of $D$. In the limit $D \rightarrow 0$, it is straightforward to solve the simplified problem (2..13)-(2..16) to find

$$
N \sim \frac{1}{(1+X)^{2}}, \quad \theta \sim 2 \log (1+X) \quad \text { as } D \rightarrow 0 .
$$

This limiting solution, in which quantum terms are everywhere negligible, agrees with the DD Debye layer solution obtained in Black et al. (2013). The dashed blue curve in Figure 2 demonstrates that numerical solutions of the full problem (2..13)-(2..16) are indeed well approximated by the DD solution (2..23) when $D$ is sufficiently small. However, the solutions for $\theta(X)$ and $N(X)$ depart significantly from the corresponding DD solutions as the value of $D$ is increased and the importance of quantum effects is magnified.

To examine the limit where $D \gg 1$, so that quantum effects dominate over space-charge variations, we perform the rescaling

$$
X=D^{1 / 4} \xi, \quad \quad N(X)=h(\xi), \quad \theta(X)=D^{1 / 2} \chi(\xi) .
$$

To leading order as $D \rightarrow \infty$, the governing equations (2..13) are transformed to

$$
\frac{1}{2 \sqrt{h}} \frac{\mathrm{d}^{2} \sqrt{h}}{\mathrm{~d} \xi^{2}}=\chi, \quad \frac{1}{2} \frac{\mathrm{d}^{2} \chi}{\mathrm{d} \xi^{2}}=-h,
$$




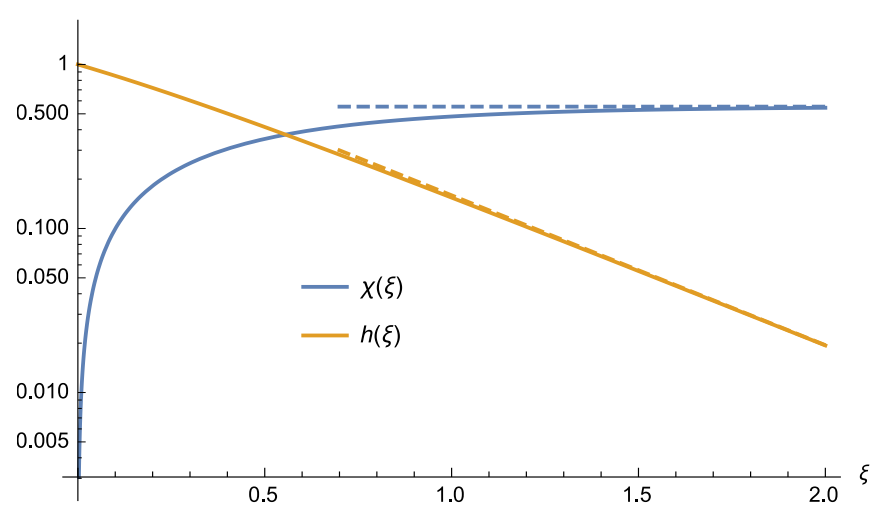

FIG. 3. The numerical solutions $\chi(\xi)$ and $h(\xi)$ of the problem (2..25)-(2..26). The dashed curves show the large- $\xi$ asymptotic behaviour (2..27).

with boundary conditions

$$
h=1, \quad \chi=0 \quad \text { at } \xi=0 .
$$

We require $h \rightarrow 0$ as $\xi \rightarrow \infty$, and we then deduce from (2..25) that $\chi$ is affine in $\xi$ as $\xi \rightarrow \infty$, which in general makes it impossible to match with (2..16). The only remaining option is to insist that $\chi$ tend to a constant as $\xi \rightarrow \infty$, and we find that there is a unique solution of (2..25) satisfying this condition, with

$$
\chi \rightarrow A, \quad h \sim B \mathrm{e}^{-2 \sqrt{2 A} \xi} \quad \text { as } \xi \rightarrow \infty,
$$

where

$$
A \approx 0.553127, \quad B \approx 1.306
$$

are numerically determined constants. The numerical solutions for $\chi(\xi)$ and $h(\xi)$ are plotted in Figure 3 , along with the asymptotic behaviour (2..27).

We note that the large- $\xi$ behaviour (2..27) does not match directly with the ultimate far-field behaviour (2..16). The behaviours may be reconciled through a complicated matching process that involves several intermediate layers. This longwinded process provides further justification for the imposition of far-field conditions (2..27), but otherwise has no bearing on the leading-order solutions plotted in Figure 3, so we omit the details here.

Having determined numerically the unique solution of the problem (2..25)-(2..27), we reverse the scalings (2..24) to obtain the leading-order large- $D$ solution of the full problem (2..18), i.e.

$$
\theta(X) \sim D^{1 / 2} \chi\left(D^{-1 / 4} X\right), \quad N(X) \sim h\left(D^{-1 / 4} X\right) \quad \text { as } D \rightarrow \infty .
$$

The dotted curves in Figure 2 show how the approximate solution (2..29) approaches numerical solutions of the full problem (2..13)-(2..16) as the value of $D$ is increased.

Figure 2 demonstrates that the solution profiles in the boundary layers at the edges of the domain may be strongly influenced by the inclusion of quantum effects. However, we note that the far-field conditions (2..16) do not depend on the quantum parameter $D$, and indeed are the same as the corresponding matching conditions obtained in Black et al. (2013) for the DD problem. Therefore the 

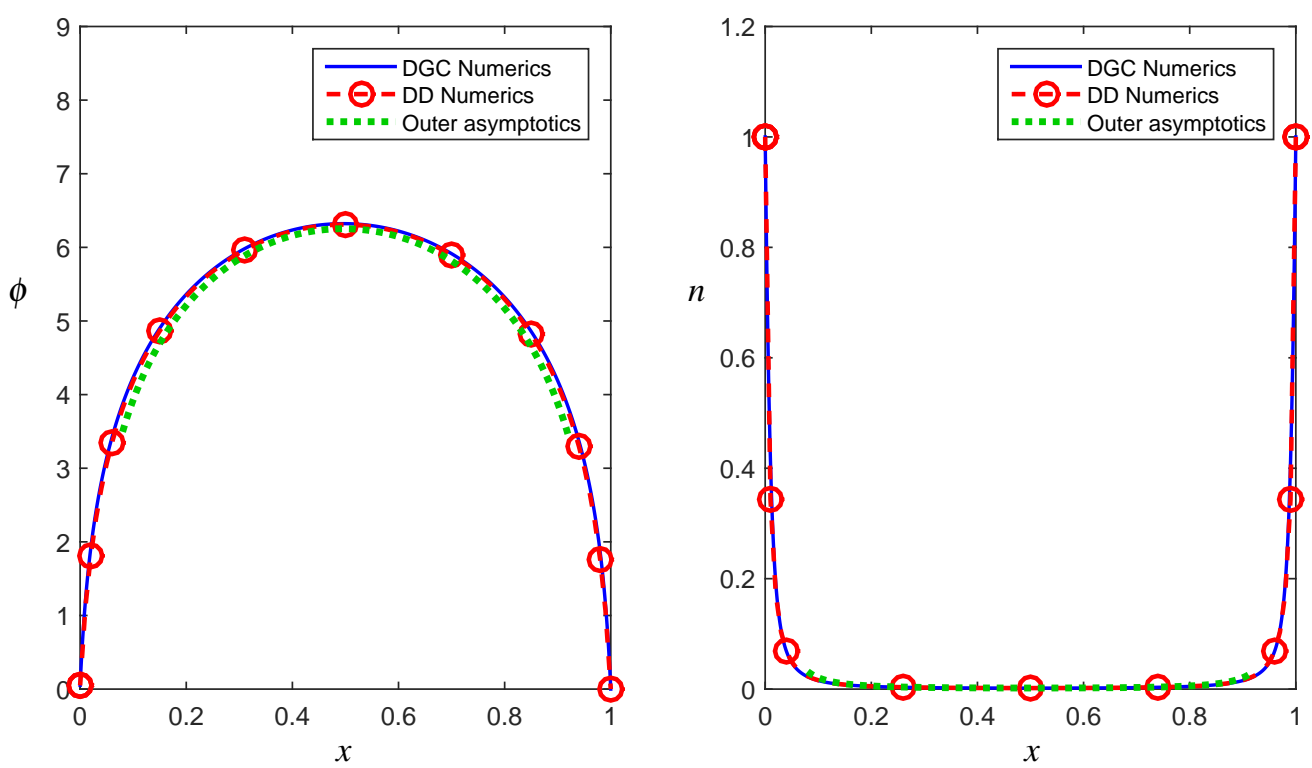

FIG. 4. Electric potential $\phi$ and electron density $n$ plotted against position $x$ with parameter values $v=0.01, \alpha=1, \delta=0.01$ and $\Phi=0.0507$

matching conditions to be applied to the outer solution (2..11), namely

$$
U(0)=0, \quad U(1)=0, \quad \frac{\mathrm{d} U}{\mathrm{~d} x}(0)=\mathrm{e}^{\Phi / 2} \sqrt{\frac{\alpha}{2}}, \quad \frac{\mathrm{d} U}{\mathrm{~d} x}(0)=-\frac{1}{\sqrt{2}},
$$

are identical to the matching conditions for the DD problem. It follows that the leading-order outer solutions for $n, \phi$ and $j$ are exactly the same as those for the DD problem.

We test this prediction in Figure 4 by plotting numerical solutions of the full DGC problem (2..1)(2..4) with illustrative parameter values $v=0.01, \alpha=1, \delta=0.01$ and $\Phi=0.0507$. We also show numerical solutions of the corresponding DD problem, where $\delta$ is set to zero, and the leading-order outer solution given by (2..10)-(2..11), where the integration constants $a, b, c$ and $w$ are determined by the boundary conditions (2..30). The three solutions are virtually indistinguishable, confirming that quantum effects have negligible influence on the outer scale.

\subsection{Large current regime}

The large current regime occurs when $j=O\left(v^{-2}\right)$ as $v \sim \delta \rightarrow 0$. Such large values of $j$ lead to correspondingly large values of the potential, with $\phi=O\left(v^{-2}\right)$ also. As shown in Black et al. (2013), 
the leading-order outer solutions in this regime are given by

$$
\phi=\frac{4 \alpha \sqrt{\mathscr{J}}}{3 v^{2}}\left[(1+\mathscr{J})^{3 / 2}-(x+\mathscr{J})^{3 / 2}\right], \quad n=\alpha \sqrt{\frac{\mathscr{J}}{x+\mathscr{J}}}
$$

where

$$
\mathscr{J}=\frac{v^{2} j}{2 \alpha^{2}}=O(1) .
$$

By setting $\phi=\Phi$ at $x=0$, we find that the applied potential is related to the normalised flux $\mathscr{J}$ by

$$
\Phi=\frac{4 \alpha \sqrt{\mathscr{J}}}{3 v^{2}}\left[(1+\mathscr{J})^{3 / 2}-\mathscr{J}^{3 / 2}\right]
$$

The outer solution (2..31) then satisfies the first two boundary conditions (2..4a) at $x=0$. It follows that both $\phi$ and $n$ are constant to leading order in the boundary layer at $x=0$, and only the second derivative of $n$ has to adjust to satisfy the the final boundary condition (2..4a) at $x=0$. This is an example of a weak boundary layer, in which quantum effects are negligible at leading order.

We therefore focus on the boundary at $x=1$. The leading-order outer solution (2..31) has the local behaviour

$$
\phi \sim \frac{2 \alpha}{v^{2}} \sqrt{\mathscr{J}(1+\mathscr{J})}(1-x), \quad n \sim \alpha \sqrt{\frac{\mathscr{J}}{1+\mathscr{J}}} \quad \text { as } x \rightarrow 1,
$$

which suggests the scalings

$$
\begin{aligned}
x & =1-\frac{v^{2 / 3} \delta^{2 / 3}}{(2 \alpha)^{1 / 3}(\mathscr{J}(1+\mathscr{J}))^{1 / 6}} \mathscr{X}, \\
n(x) & =\alpha \sqrt{\frac{\mathscr{J}}{1+\mathscr{J}} \mathscr{N}(\mathscr{X}),} \\
\phi(x) & =\frac{2 \alpha}{v^{2}} \sqrt{\mathscr{J}(1+\mathscr{J})(1-x)+\frac{\alpha^{1 / 3} \mathscr{J}^{1 / 6} \delta^{4 / 3}}{2^{2 / 3}(1+\mathscr{J})^{5 / 6} v^{2 / 3}} \psi(\mathscr{X}) .}
\end{aligned}
$$

By substituting (2..35) into the governing equations (2..1)-(2..4) and then letting $v \rightarrow 0$, we obtain the leading-order inner equation

$$
\frac{\mathrm{d}}{\mathrm{d} \mathscr{X}}\left(\frac{1}{\sqrt{\mathscr{N}}} \frac{\mathrm{d}^{2} \sqrt{\mathscr{N}}}{\mathrm{d} \mathscr{X}^{2}}\right)=\frac{\mathscr{N}-1}{\mathscr{N}}
$$

with boundary and matching conditions

$$
\begin{aligned}
& \mathscr{N}=\frac{1}{\alpha} \sqrt{\frac{1+\mathscr{J}}{\mathscr{J}}}, \quad \frac{\mathrm{d}^{2} \sqrt{\mathscr{N}}}{\mathrm{d} \mathscr{X}^{2}}=0 \quad \text { at } \quad \mathscr{X}=0, \\
& \mathscr{N}(\mathscr{X}) \rightarrow 1 \quad \text { as } \quad \mathscr{X} \rightarrow \infty .
\end{aligned}
$$

The perturbation $\psi(\mathscr{X})$ to the potential in the boundary layer may in principle be determined a posteriori from the decoupled equation

$$
\frac{\mathrm{d}^{2} \psi}{\mathrm{d} \mathscr{X}^{2}}=-\mathscr{N}
$$




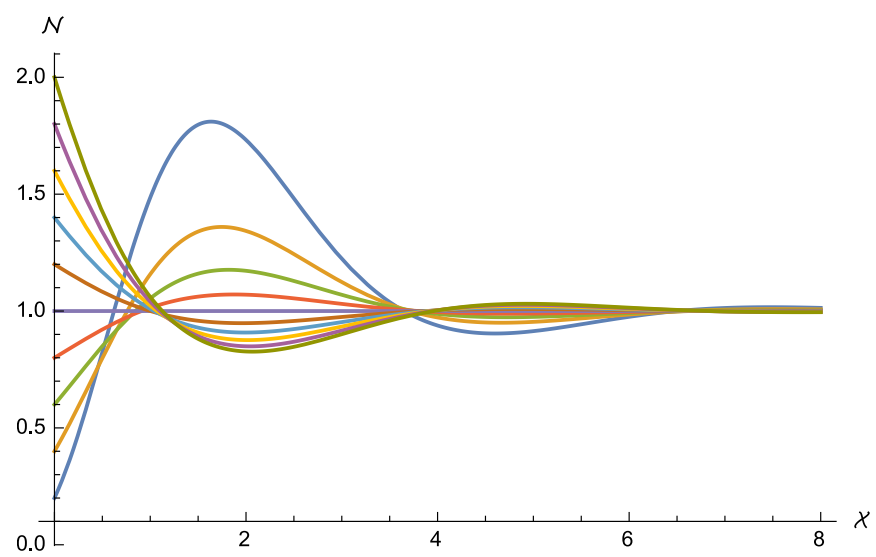

FIG. 5. Normalised electron density $\mathscr{N}(\mathscr{X})$ plotted versus normalised distance $\mathscr{X}$ in the boundary layer at $x=1$, with $\mathscr{N}(0)=$ $0.2,0.4, \cdots, 2.0$.

We solve (2..36)-(2..37) numerically by shooting from $\mathscr{X} \rightarrow \infty$. To determine an initial condition for the shooting method we linearise about $\mathscr{N}=1$ to find that

$$
\mathscr{N} \sim 1+C \mathrm{e}^{-\hat{\mathscr{X}} / 2^{2 / 3}} \cos \left(\sqrt{3} \hat{\mathscr{X}} / 2^{2 / 3}\right) \quad \text { as } \hat{\mathscr{X}} \rightarrow \infty,
$$

where $C$ is a constant and $\hat{\mathscr{X}}$ is a translation of $\mathscr{X}$ given by $\hat{X}=\mathscr{X}-\mathscr{X}^{*}$. For a given value of $C$, we use (2..39) to solve (2..36) as an initial-value proble, starting from $\hat{\mathscr{X}} \gg 1$. We shoot until the condition

$$
\frac{\mathrm{d}^{2} \sqrt{\mathscr{N}}}{\mathrm{d} \hat{\mathscr{X}}^{2}}=0 \quad \text { at } \hat{\mathscr{X}}=\mathscr{X}^{*}
$$

is satisfied, read off the corresponding value of $\hat{\mathscr{X}}=\mathscr{X}^{*}$ where this occurs, and thus find

$$
\left.\mathscr{N}\right|_{\hat{X}=\mathscr{X}^{*}}=\frac{1}{\alpha} \sqrt{\frac{1+\mathscr{J}}{\mathscr{J}}} .
$$

The value of the shooting parameter $C$ is thus determined by the values of $\alpha$ and $\mathscr{J}$.

In Figure 5, we plot numerical solutions for $\mathscr{N}(\mathscr{X})$ obtained using this procedure for different values of $\mathscr{N}(0)$. We note that $\mathscr{N}(\mathscr{X}) \equiv 1$ is an exact solution of the problem (2..36)-(2..37) in the special case where $\mathscr{J}=1 /\left(\alpha^{2}-1\right)$. For this particular value of the flux, the outer solution (2..31) exactly satisfies the boundary condition $n(1)=1$, so only a weak boundary layer is required. For any other value of $\mathscr{J}$, the density profile exhibits oscillations, whose amplitude grows the further $\mathscr{N}(0)$ departs from unity. These oscillations are a distinctive new feature exhibited by solutions of the DGC model but never observed in the absence of quantum effects.

In Figure 6 we compare solutions of the boundary layer shooting problem described in this section with numerical solutions of the full DGC model and of the DD model. The DGC and DD solutions for the potential $\phi(x)$ are so close as to be indistinguishable everywhere, reflecting the fact that the quantum 

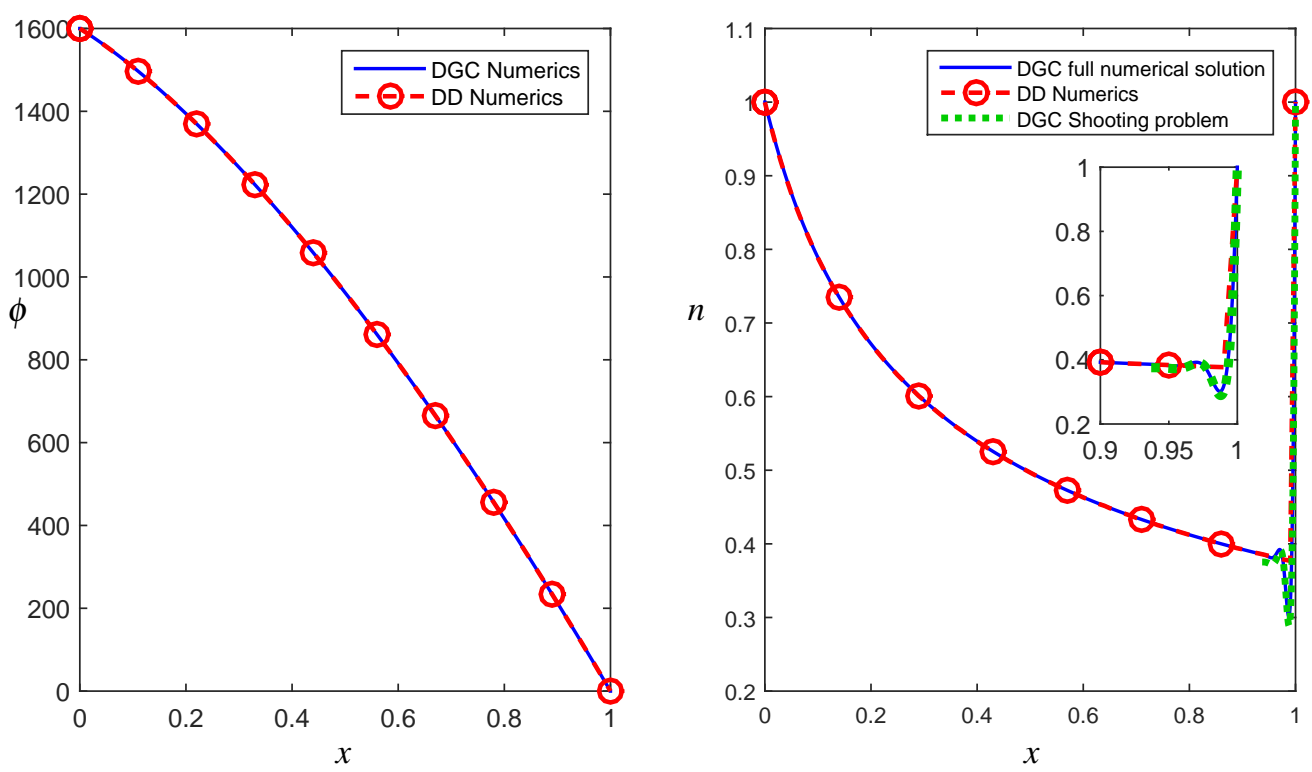

FIG. 6. Electric potential $\phi$ and electron density $n$ plotted against position $x$ where $\Phi=1600, v=0.02, \alpha=1$ and $\delta=0.02$.

term $\psi(\mathscr{X})$ is always a small correction to the leading term in the expansion $(2 . .35 \mathrm{c})$. However, the electron density profile is profoundly altered by the inclusion of quantum effects, which result in large oscillations close to $x=1$ not produced by the DD calculations. These oscillations are, however, captured extremely well by the boundary layer solution constructed using the shooting method described above. Nevertheless, as in $\S 2.3$, the solutions on the outer scale are not significantly affected by the inclusion of quantum effects.

\subsection{Resistance}

The DD and DGC models in principle allow us to predict the variations in the electron density $n$ and electric potential $\phi$ across the glass layer. However, the only easily measurable quantity is the net contact resistance $R$, defined as in Black et al. (2013) by

$$
R=\frac{\Phi+\log \alpha}{j}=\int_{0}^{1} \frac{\mathrm{d} x}{n(x)} .
$$

As shown above in $\S \S 2.3 .-2.4$., provided $v \sim \delta \ll 1$, the leading-order outer solution for $n(x)$ is unaffected by the inclusion of quantum effects. Therefore we predict that the net resistance of the layer is not significantly altered when the quantum term is included, so that quantum effects are effectively unmeasurable on the macroscopic scale.

This prediction is confirmed in Figure 7, where we plot resistance $R$ versus electron flux $j$ for illustrative parameter values $v=0.01, \alpha=1$ and $\delta=0.01$. The red solid curve shows the the results of 


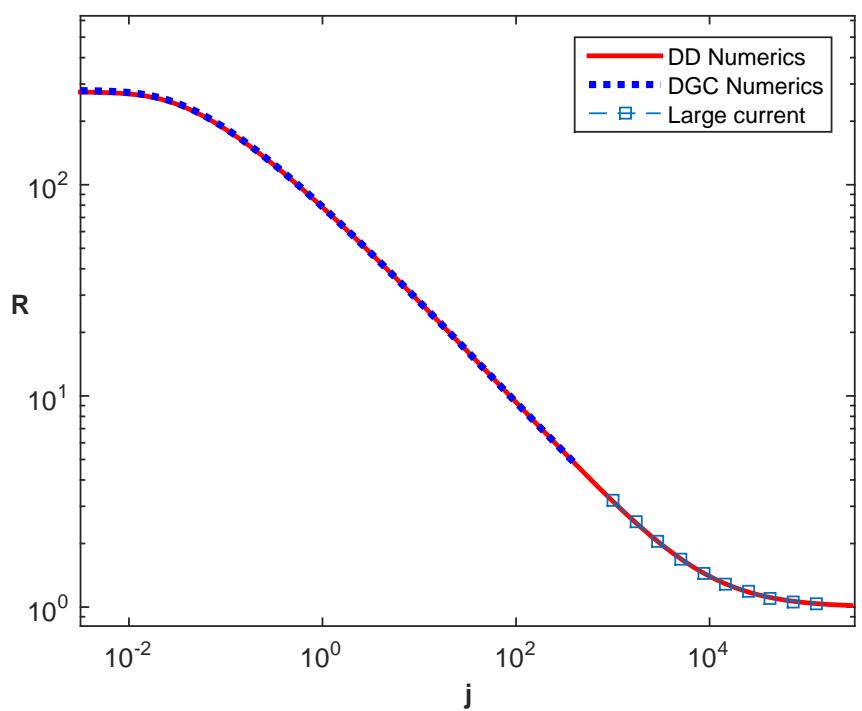

FIG. 7. Resistance $R$ plotted against electron flux $j$ with $v=0.01, \alpha=1$ and $\delta=0.01$ : numerical solution of DD model [red]; numerical solution of DGC model [blue dotted]; large current approximation (2..43) [blue squares].

DD numerical calculations, and the blue dotted curve shows the corresponding DGC numerical results. We observe that the curves are practically indistinuishable as $j$ varies over many orders of magnitude. For $j \gtrsim 10^{2}$, the DGC numerics becomes prohibitively stiff. However, we plot also, using squares, the resistance predicted by the large current result (2..33), namely

$$
R \sim \frac{2 \sqrt{2}}{3 v \sqrt{j}}\left[\left(1+\frac{v^{2} j}{2 \alpha^{2}}\right)^{3 / 2}-\left(\frac{v^{2} j}{2 \alpha^{2}}\right)^{3 / 2}\right]
$$

which we recall is valid in the limit $j=O\left(v^{-2}\right)$ and $v \sim \delta \rightarrow 0$. Figure 7 shows that (2..43) gives an excellent approximation of the variation of resistance with electron flux for $j \gtrsim 10^{2}$.

\section{Density Gradient Tunnelling theory}

\subsection{Model formulation}

In this section, by extending the work of Ancona (1990), we investigate DGT theory for modelling charge transport in very small devices (1-3 nm). DGT theory is a continuum alternative to microscopic quantum transport theories for modelling quantum tunnelling. A schematic of the device to be analysed is shown in Figure 8. To formulate the mathematical model for charge transport through an insulator with identical metal contacts we start from the system (1..2) and make the following assumptions.

- Only electron tunnelling is important and therefore hole tunnelling is neglected.

- The system is in steady state and the electron tunnelling is one-dimensional. 


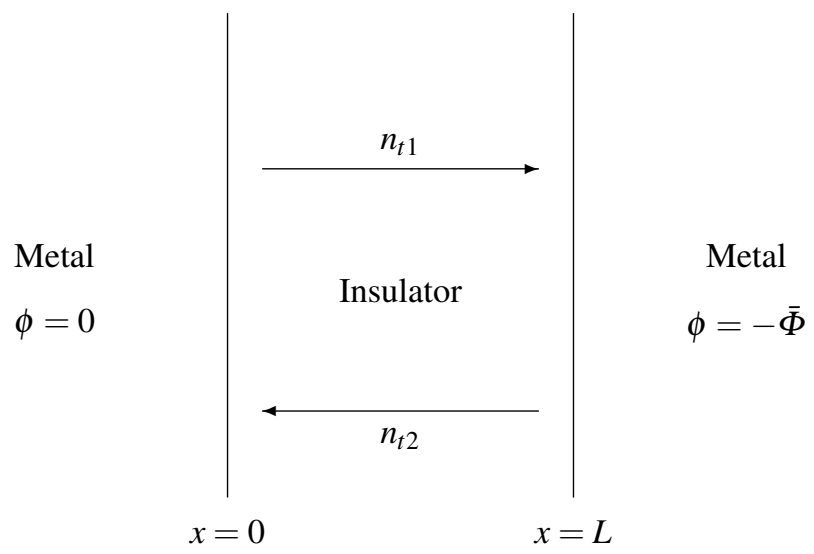

FIG. 8. Schematic of the problem modelled using DGT theory.

- Diffusion is negligible.

We thus arrive at the following system of equations:

$$
\frac{\mathrm{d}^{2} \phi}{\mathrm{d} x^{2}}=-\frac{q n_{t}}{\varepsilon}, \quad \frac{\mathrm{d} J}{\mathrm{~d} x}=\frac{\mathrm{d} \Psi}{\mathrm{d} x}=0,
$$

where $J=n_{t} v_{n}$ is the tunnelling electron flux and

$$
\Psi=-\phi+\frac{\hbar^{2}}{2 m_{n} r_{n} q} \frac{1}{\sqrt{n_{t}}} \frac{\mathrm{d}^{2} \sqrt{n_{t}}}{\mathrm{~d} x^{2}}-\frac{m_{n}}{2 q} \frac{J^{2}}{n_{t}^{2}} .
$$

The kinetic electrochemical potential $\Psi$ is thus constant throughout the insulator and dependent on the electrode from which the electrons originate. To resolve the contradiction that $\Psi$ is constant but takes different values on the two electrodes, Ancona (1990) defines two distinct electron populations inside the the insulator. As shown in Figure 8, we have electron population 1 with density $n_{t 1}$ moving from left to right, and electron population 2 with density $n_{t 2}$ moving from right to left. The two populations of electrons satisfy "upstream" and "downstream" boundary conditions respectively.

We now summarise the boundary conditions applied; more details are given in Ancona \& Svizhenko (2008). At each electrode a constant upstream electron density is specified along with a boundary condition to ensure continuity of the kinetic electrochemical potential across the metal-insulator interface:

$$
\begin{array}{ll}
n_{t 1}=n_{M}, & \theta_{\mathrm{MB}}=\frac{\hbar^{2}}{2 m_{n} r_{n} q} \frac{1}{\sqrt{n_{t 1}}} \frac{\mathrm{d}^{2} \sqrt{n_{t 1}}}{\mathrm{~d} x^{2}}-\frac{m_{n}}{2 q} \frac{J_{1}^{2}}{n_{t 1}^{2}} \quad \text { at } \quad x=0, \\
n_{t 2}=n_{M}, & \theta_{\mathrm{MB}}=\frac{\hbar^{2}}{2 m_{n} r_{n} q} \frac{1}{\sqrt{n_{t 2}}} \frac{\mathrm{d}^{2} \sqrt{n_{t 2}}}{\mathrm{~d} x^{2}}-\frac{m_{n}}{2 q} \frac{J_{2}^{2}}{n_{t 2}^{2}} \quad \text { at } \quad x=L,
\end{array}
$$

where

$$
\theta_{\mathrm{MB}}=\frac{W_{\text {electrode }}+\chi_{\text {ins }}}{q}
$$


is the "Schottky barrier height", $W_{\text {electrode }}$ is the workfunction of the metal electrode and $\chi_{\text {ins }}$ is the electron affinity of the insulator. We will consider identical contacts so that the Schottky barrier height is the same at each electrode. The constant $n_{M}$ in (3..3) is the tunnelling electron density at the metalinsulator interface.

We also impose boundary conditions on the downstream electron population as follows:

$$
\begin{aligned}
& J_{1}=-\gamma n_{t 1} \quad \text { at } x=L, \quad J_{2}=\gamma n_{t 2} \quad \text { at } x=0, \\
& \frac{\mathrm{d} n_{t 1}}{\mathrm{~d} x}=0 \quad \text { at } x=L, \quad \frac{\mathrm{d} n_{t 2}}{\mathrm{~d} x}=0 \quad \text { at } x=0 .
\end{aligned}
$$

Here $J_{1,2}$ is the flux of each population, determined by the relevant tunnelling density and the average electron velocity $\gamma$ at the downstream electrode, which is used as a fitting parameter. Ancona (2011) attributes the boundary conditions (3..6) to "elastic capture" of the tunnelling electrons at the downstream contact.

Finally, the boundary conditions are completed by specifying the potential difference applied across the insulator

$$
\phi=0 \quad \text { at } x=0, \quad \phi=-\bar{\Phi} \quad \text { at } x=L .
$$

Combining the continuity equations (3..1)-(3..2) for each electron population and making use of the boundary conditions (3..3) and (3..7) we obtain the system of equations

$$
\begin{aligned}
& \frac{\mathrm{d}^{2} \phi}{\mathrm{d} x^{2}}=-\frac{q\left(n_{t 1}+n_{t 2}\right)}{\varepsilon}, \\
&-\phi-\theta_{\mathrm{MB}}+\frac{\hbar^{2}}{2 m_{n} r_{n} q} \frac{1}{\sqrt{n_{t 1}}} \frac{\mathrm{d}^{2} \sqrt{n_{t 1}}}{\mathrm{~d} x^{2}}-\frac{m_{n}}{2 q} \frac{J_{1}^{2}}{n_{t 1}^{2}}=0, \\
&-\phi-\left(\theta_{\mathrm{MB}}+V\right)+\frac{\hbar^{2}}{2 m_{n} r_{n} q} \frac{1}{\sqrt{n_{t 2}}} \frac{\mathrm{d}^{2} \sqrt{n_{t 2}}}{\mathrm{~d} x^{2}}-\frac{m_{n}}{2 q} \frac{J_{2}^{2}}{n_{t 2}^{2}}=0,
\end{aligned}
$$

with boundary conditions

$$
\begin{aligned}
& \phi=0, \quad n_{t 1}=n_{M}, \quad \frac{\mathrm{d} n_{t 2}}{\mathrm{~d} x}=0, \quad J_{2}=\gamma n_{t 2} \quad \text { at } x=0, \\
& \phi=-\bar{\Phi}, \quad n_{t 2}=n_{M}, \quad \frac{\mathrm{d} n_{t 1}}{\mathrm{~d} x}=0 \quad J_{1}=-\gamma n_{t 1} \quad \text { at } x=L,
\end{aligned}
$$

where $J_{1}$ and $J_{2}$ are the constant electron fluxes,

We now introduce the following nondimensional variables

$$
\begin{aligned}
& \phi=\frac{\gamma^{2} m_{n}}{2 q} \phi^{*}, \quad n_{t 1}=n_{M} n_{t 1}^{*}, \quad n_{t 2}=n_{M} n_{t 2}^{*} \\
& x=\frac{L}{2}\left(1+x^{*}\right), \quad J_{1}=\gamma n_{M} J_{1}^{*}, \quad J_{2}=\gamma n_{M} J_{2}^{*} .
\end{aligned}
$$

Substituting (3..10) into (3..8)-(3..9) and dropping the starred notation, we obtain the dimensionless 


\begin{tabular}{ccccc} 
Parameter & Symbol & Value & Units & Notes \\
\hline Glass layer thickness & $L$ & $2 \times 10^{-9}$ & $\mathrm{~m}$ & \\
Statistical factor & $r_{n}$ & 1 & - & $(1)$ \\
Interfacial electron density & $n_{M}$ & $\ll 10^{25}$ & $\mathrm{~m}^{-3}$ & $(2)$ \\
Downstream electron velocity & $\gamma$ & $10^{6}$ & $\mathrm{~m} \mathrm{~s}^{-1}$ & $(3)$ \\
Schottky barrier height & $\theta_{\mathrm{MB}}$ & 4 & $\mathrm{~V}$ & $(4)$
\end{tabular}

Table 2. Typical values of physical parameters for DGT model. (1) This value corresponds to the relevant low electron density limit (see Ancona (1990)). (2) The exact size of $n_{M}$ is unknown but it is expected to be small (see Ancona (1990)). (3) Ancona (1990) estimated the size of $\gamma$ by matching numerical solutions of (3..11)-(3..12) to experiments. (4) For example the workfunction of silver is $4.3 \mathrm{eV}$ (see Sze \& Ng (2006)).

equations

$$
\begin{aligned}
& v_{\mathrm{QM}}^{2} \frac{\mathrm{d}^{2} \phi}{\mathrm{d} x^{2}}=-\left(n_{t 1}+n_{t 2}\right), \\
&-\left(\phi+\Theta_{\mathrm{MB}}\right)+\frac{B}{\sqrt{n_{t 1}}} \frac{\mathrm{d}^{2} \sqrt{n_{t 1}}}{\mathrm{~d} x^{2}}-\frac{J_{1}^{2}}{n_{t 1}^{2}}=0, \\
&-\phi-\left(\Theta_{\mathrm{MB}}+V\right)+\frac{B}{\sqrt{n_{t 2}}} \frac{\mathrm{d}^{2} \sqrt{n_{t 2}}}{\mathrm{~d} x^{2}}-\frac{J_{2}^{2}}{n_{t 2}^{2}}=0,
\end{aligned}
$$

and boundary conditions

$$
\begin{aligned}
& \phi=0, \quad n_{t 1}=1, \quad \frac{\mathrm{d} n_{t 2}}{\mathrm{~d} x}=0, \quad J_{2}=n_{t 2} \quad \text { at } x=-1, \\
& \phi=-V, \quad n_{t 2}=1, \quad \frac{\mathrm{d} n_{t 1}}{\mathrm{~d} x}=0, \quad J_{1}=-n_{t 1} \quad \text { at } x=1 .
\end{aligned}
$$

The dimensionless parameters are

$$
\Theta_{\mathrm{MB}}=\frac{2 q \theta_{\mathrm{MB}}}{\gamma^{2} m_{n}}, \quad V=\frac{2 q \bar{\Phi}}{\gamma^{2} m_{n}}, \quad B=\frac{4 \hbar^{2}}{L^{2} \gamma^{2} m_{n}^{2} r_{n}}, \quad v_{\mathrm{QM}}=\sqrt{\frac{2 \varepsilon \gamma^{2} m_{n}}{q^{2} L^{2} n_{M}}} .
$$

The parameter $v_{\mathrm{QM}}$ is the scaled Debye length and $\sqrt{B}$ is the electron quantum length. The parameters $v$ and $\delta$ in $\S 2$. have the same physical interpretation but a different form, since, here we scale the electric potential with the inertial effects rather than with diffusion. We estimate the sizes of these parameters using the typical physical properties listed in Tables 1 and 2. The size of $\gamma$ estimated by Ancona (1990) ensures that $\Theta_{\mathrm{MB}}$ and $V$ are both $O(1)$, while $B$ is $O\left(10^{-2}\right)$. Finally, the small device length $L$ and the anticipated low tunnelling electron density $n_{M}$ suggest that $v_{\mathrm{QM}} \gg 1$, and we can therefore approximate the dimensionless potential using

$$
\phi \sim-\frac{V}{2}(x+1) .
$$

Substituting (3..14) into (3..11b)-(3..11c) and defining $\sqrt{n_{t 1}}=s_{1}, \sqrt{n_{t 2}}=s_{2}$ we obtain the equations

$$
\frac{B}{V s_{j}} \frac{\mathrm{d}^{2} s_{j}}{\mathrm{~d} x^{2}}=\frac{J_{j}^{2}}{V s_{j}^{4}}-\frac{1}{2}\left(x-z_{j}\right), \quad j=1,2,
$$


where

$$
z_{1}=\frac{2 \Theta_{\mathrm{MB}}}{V}-1
$$

$$
z_{2}=\frac{2 \Theta_{\mathrm{MB}}}{V}+1=z_{1}+2
$$

The boundary conditions now take the form

$$
\begin{aligned}
& s_{1}=1, \quad \frac{\mathrm{d} s_{2}}{\mathrm{~d} x}=0, \quad J_{2}=s_{2}^{2} \quad \text { at } x=-1, \\
& s_{2}=1, \quad \frac{\mathrm{d} s_{1}}{\mathrm{~d} x}=0, \quad J_{1}=-s_{1}^{2} \quad \text { at } x=1 .
\end{aligned}
$$

The system (3..15)-(3..17) is the nondimensional analogue of the model (4.2), (3.2c) and (3.4) in Ancona Ancona (1990). We will now exploit the smallness of the parameter $B$ to conduct asymptotic analysis on the system (3..15)-(3..17) and hence estimate how the fluxes $J_{1}$ and $J_{2}$ depend on the parameters $B, V$ and $\Theta_{\mathrm{MB}}$. The qualitative behaviour of solutions of equation (3..15) depends crucially on whether $z_{j}$ lies in the interval $(-1,1)$. We find that $z_{2}>1$ for physical values of $\Theta_{\mathrm{MB}}$ and $V$; however, $z_{1}>1$ when $V<\Theta_{\mathrm{MB}}$ and $z_{1} \in(-1,1)$ when $V>\Theta_{\mathrm{MB}}$, and we will show that the solution for $s_{1}(x)$ has very different qualitative behaviour in the two cases.

\subsection{Numerical solution}

To validate the asymptotic analysis performed below, we also solve the problem (3..15)-(3..17) numerically. To find the numerical solution for $s_{1}$ we begin by defining

$$
s_{1}(x)=\frac{\sqrt{-J_{1}}}{V^{1 / 4}} \omega(x) .
$$

Substituting (3..18) into (3..15)-(3..17) we obtain the shooting problem

$$
\frac{B}{V} \omega^{\prime \prime}=\frac{1}{\omega^{3}}+\frac{\left(z_{1}-x\right)}{2} \omega
$$

with

$$
\omega^{\prime}(1)=0, \quad \omega(1)=V^{1 / 4} .
$$

Given the values of $B$ and $V$, we solve (3..19) numerically as an initial-value problem, starting from $x=1$. The electron flux is then found a posteriori using

$$
J_{1}=-\frac{\sqrt{V}}{\omega(-1)^{2}} .
$$

The numerical solution for $s_{2}$ is found by solving an analagous shooting problem starting from $x=-1$.

\subsection{Normal tunnelling: $V<\Theta_{M B}$}

In the "normal tunnelling" regime, where $V<\Theta_{\mathrm{MB}}$, the final term in (3..15) is negative throughout the domain $x \in[-1,1]$ for both electron populations $j=1,2$. In this section we use boundary layer and WKBJ asymptotic analysis to approximate solutions of the problem (3..15)-(3..17) in the physically relevant limit where $B \ll 1$. 
First considering the left-moving electrons (population 1), we hypothesise that the flux $J_{1}$ is negative and exponentially small in the parameter $B$. We therefore let $J_{1}=-\varepsilon_{1}^{2}$, where $\varepsilon_{1}=O\left(\mathrm{e}^{-1 / \sqrt{B}}\right)$. The boundary conditions (3..17) imply that $s_{1}=O\left(\varepsilon_{1}\right)$ near $x=1$, and we therefore solve for a boundary layer in this region. We let $s_{1}=\varepsilon_{1} S$ and $x=1-\sqrt{B} \tilde{X}$ in equations (3..15)-(3..17) before letting $B \rightarrow 0$ to find the leading-order boundary layer problem

$$
\frac{\mathrm{d}^{2} S}{\mathrm{~d} \tilde{X}^{2}}=\frac{1}{S^{3}}+m^{2} S . \quad S(0)=1, \quad \frac{\mathrm{d} S}{\mathrm{~d} \tilde{X}}=0,
$$

where

$$
m=\sqrt{\frac{V\left(z_{1}-1\right)}{2}}
$$

The problem (3..21a) has the solution

$$
S=\sqrt{\cosh ^{2}(m \tilde{X})+\frac{1}{m^{2}} \sinh ^{2}(m \tilde{X})} .
$$

To determine the outer solution away from the boundary layer at $x=1$ we apply the WKBJ method. By seeking an asymptotic expansion for $s_{1}(x)$ in the form $s_{1} \sim A(x) \mathrm{e}^{f(x) / \sqrt{B}}$, we find the leading-order outer approximation

$$
s_{1} \sim\left(\frac{z_{1}+1}{z_{1}-x}\right)^{1 / 4} \exp \left[-\frac{\sqrt{2 V}}{3 \sqrt{B}}\left(\left(z_{1}+1\right)^{3 / 2}-\left(z_{1}-x\right)^{3 / 2}\right)\right] .
$$

By matching the inner solution (3..23) to the outer solution (3..24) we find

$$
\varepsilon_{1} \sim \frac{2 m}{\sqrt{m^{2}+1}}\left(\frac{z_{1}+1}{z_{1}-1}\right)^{1 / 4} \exp \left[-\frac{\sqrt{2 V}}{3 \sqrt{B}}\left(\left(z_{1}+1\right)^{3 / 2}-\left(z_{1}-1\right)^{3 / 2}\right)\right]
$$

and we therefore obtain the following approximation for the left moving flux

$$
J_{1} \sim-\frac{4 \sqrt{\Theta_{\mathrm{MB}}\left(\Theta_{\mathrm{MB}}-V\right)}}{1+\Theta_{\mathrm{MB}}-V} \exp \left[-\frac{8}{3 V \sqrt{B}}\left(\Theta_{\mathrm{MB}}^{3 / 2}-\left(\Theta_{\mathrm{MB}}-V\right)^{3 / 2}\right)\right] \text {. }
$$

Now considering the right moving electrons (population 2), we follow an analagous procedure to find in the boundary layer near $x=-1$

$$
\frac{s_{2}}{\varepsilon_{2}} \sim \sqrt{\cosh ^{2}(\hat{m} \hat{X})+\frac{1}{\hat{m}^{2}} \sinh ^{2}(\hat{m} \hat{X})}
$$

where $J_{2}=\varepsilon_{2}^{2}, \hat{m}=\sqrt{V\left(z_{2}+1\right) / 2}$ and $\hat{X}=(x+1) / \sqrt{B}$. Similarly, a WKBJ approach gives the leadingorder outer solution as

$$
s_{2} \sim\left(\frac{z_{2}-1}{z_{2}-x}\right)^{1 / 4} \exp \left[-\frac{\sqrt{2 V}}{3 \sqrt{B}}\left(\left(z_{2}-x\right)^{3 / 2}-\left(z_{2}-1\right)^{3 / 2}\right)\right],
$$



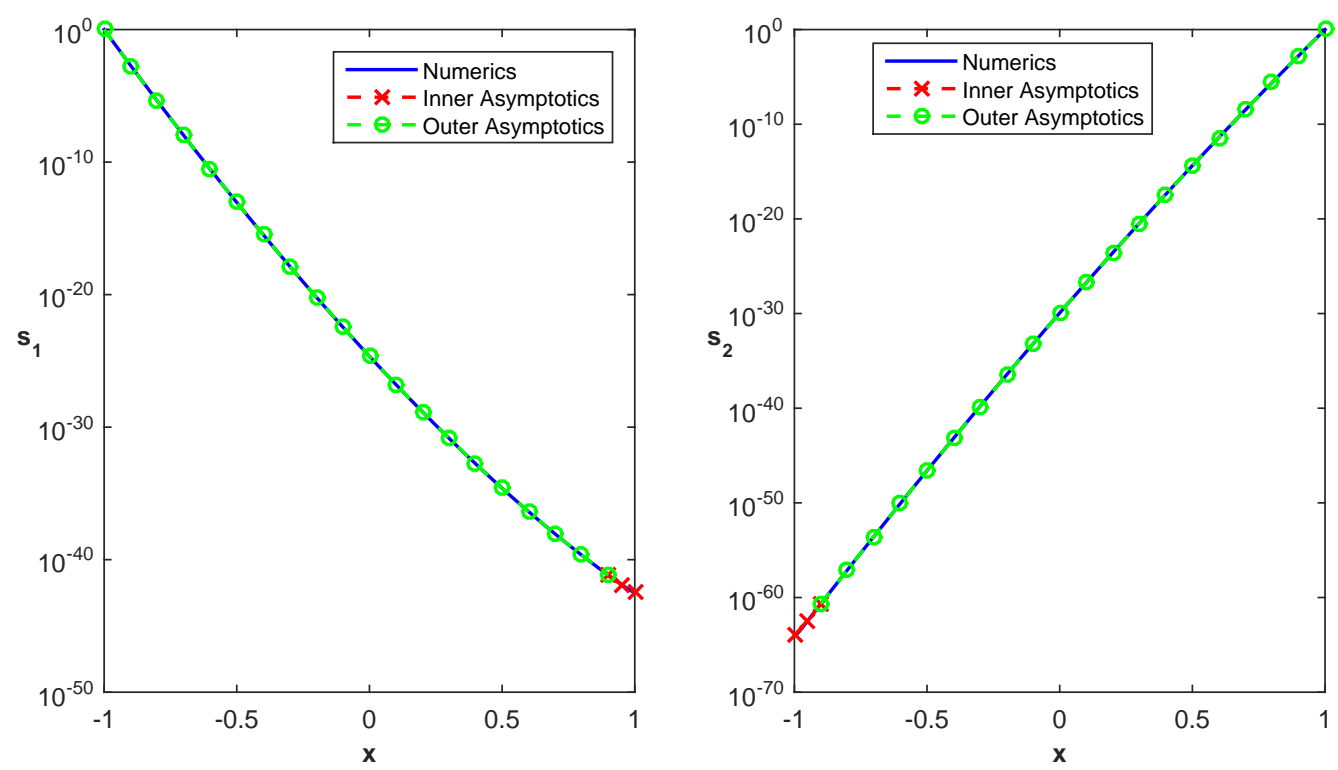

FIG. 9. Square rooted electron populations $s_{1}$ and $s_{2}$ plotted against position $x$ with parameter values $B=10^{-3}, V=3, \Theta_{\mathrm{MB}}=4$ and hence $z_{1}=5 / 3, z_{2}=11 / 3$. We show numerical solutions of the full problem (3..15)-(3..17) [solid blue]; the boundary layer solutions (3..23) and (3..27) [red crosses]; the WKBJ outer solutions (3..24) and (3..28) [green circles].

and by matching the inner and outer solutions, we estimate the right moving flux as

$$
J_{2} \sim \frac{4 \sqrt{\Theta_{\mathrm{MB}}\left(\Theta_{\mathrm{MB}}+V\right)}}{1+\Theta_{\mathrm{MB}}+V} \exp \left[-\frac{8}{3 V \sqrt{B}}\left(\left(\Theta_{\mathrm{MB}}+V\right)^{3 / 2}-\Theta_{\mathrm{MB}}^{3 / 2}\right)\right] .
$$

In Figure 9, we demonstrate the excellent agreement between the asymptotic expressions (3..23), (3..24), (3..27), (3..28) and the numerical solution of the full problem (3..15)-(3..17), using illustrative parameter values $B=10^{-3}, V=3$ and $\Theta_{\mathrm{MB}}=4$. The asymptotic approximations for the fluxes (3..26) and (3..29) give $J_{1} \sim-1.41 \times 10^{-85}$ and $J_{2} \sim 9.89 \times 10^{-129}$ for these parameter values, which are very close to the numerically calculated values $J_{1} \approx-1.40 \times 10^{-85}$ and $J_{2} \approx 9.89 \times 10^{-129}$. The close agreement between asymptotics and numerics both validates our asymptotic analysis and demonstrates the success of the shooting method described in $\S 3.2$. in accurately computing the exponentially small fluxes.

It is easy to see from the asymptotic expressions (3..26) and (3..29) that $J_{2} /\left|J_{1}\right|$ is exponentially small as $B \rightarrow 0$ with $V<\Theta_{\mathrm{MB}}$. Therefore the leading-order approximation for the total electron flux $J=J_{1}+J_{2}$ is simply $J \sim J_{1}$, with $J_{1}$ given by equation (3..26). We test this prediction in Figure 10 by plotting the variation of the total current density $(-J)$ with applied potential $V$ with parameter values $B=10^{-3}$ and $\Theta_{\mathrm{MB}}=4$. The approximation (3..26) and the results of numerical solution of the shooting problem (3..19) are indistinguishable, and $J$ varies by over 30 orders of magnitude as $V$ increases from 0.1 to $\Theta_{\mathrm{MB}}$. Our asymptotic prediction (3..26) implies that either increasing the length of the insulator (decreasing $B$ ), or increasing the barrier height $\Theta_{\mathrm{MB}}$, or decreasing the applied potential difference $V$, would cause an exponential increase in the current density, all of which are well-known features of 


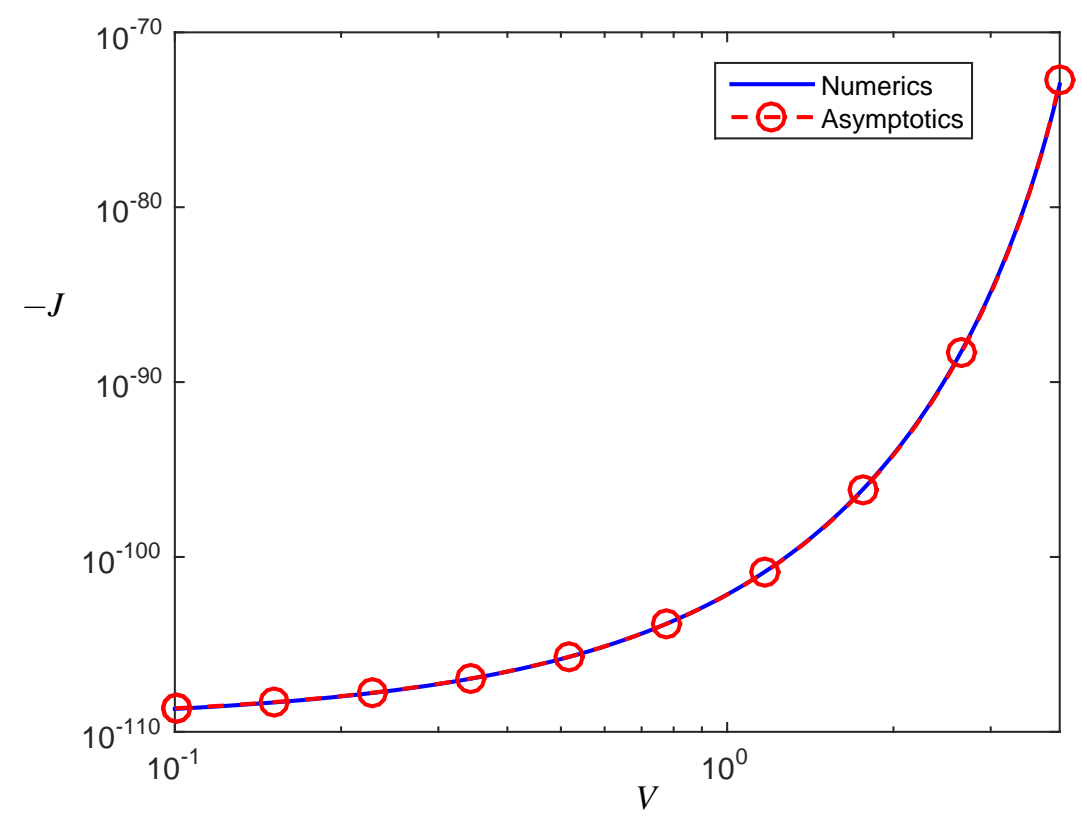

FIG. 10. Current density $(-J)$ plotted against applied potential difference $V$ with parameter values $B=10^{-3}$ and $\Theta_{\mathrm{MB}}=4$. The blue solid curve shows the results of numerical solution of the shooting problem (3..19). The red circles curve shows the leading-order asymptotic solution $J \sim J_{1}$, with $J_{1}$ given by equation (3..26).

quantum mechanical tunnelling Simmons (1963). Finally, we note that Ancona (1990) did find the outer WKBJ solutions (3..24), (3..28) but did not match to the correct inner boundary layer problem and therefore did not obtain our accurate approximation (3..26) to the total current density.

\subsection{Fowler-Nordheim tunnelling: $V>\Theta_{M B}$}

\section{4..1 Introduction}

In the "Fowler-Nordheim tunnelling" regime, where $V>\Theta_{\mathrm{MB}}$, equation (3..16) implies that $z_{2}>1$ but $z_{1} \in(-1,1)$. Therefore the solution (3..29) for $J_{2}$ still holds in this regime, but the solution (3..26) for $J_{1}$ is no longer valid, because the final term in equation (3..15) changes sign within the domain, leading to different qualitative behaviour in the two regions $-1<x<z_{1}$ and $z_{1}<x<1$. In this section we perform an asymptotic analysis of this regime in the limit $B \rightarrow 0$, by constructing leading-order solutions in $x<z_{1}$ and $x>z_{1}$ and then matching them together across an inner layer at $x=z_{1}$. The analysis is facilitated by returning to the notation (3..18) introduced in $\S 3.2$.. Thus we solve for the scaled electron density $\omega(x)$ which satisfies the initial-value problem (3..19) and then evaluate the flux $J_{1}$ using equation (3..20). 


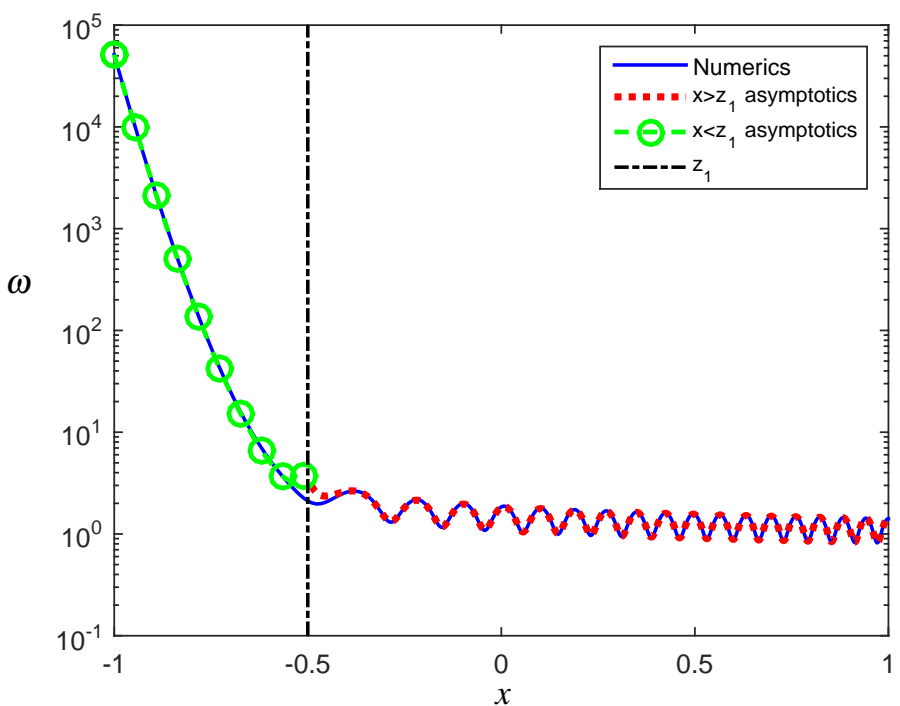

FIG. 11. The scaled square rooted electron population $\omega$ plotted against position $x$ with parameter values $B=10^{-3}, V=4$, $\Theta_{\mathrm{MB}}=1$, and hence $z_{1}=-1 / 2$ (indicated by a black dashed curve). The solid blue curve shows the numerical solution; the red dotted curve shows the asymptotic oscillatory solution (3..46) in $x>z_{1}$; the green circles show the asymptotic exponential solution (3..48) in $x<z_{1}$, with $K \approx 1.142$.

\section{4..2 Oscillatory solution: $z_{1}<x<1$}

First we consider the region $z_{1}<x<1$, where the final term in equation (3..19a) is negative, and we therefore anticipate that the solution displays fast oscillations when $B \ll 1$. We use the method of multiple scales, with the short length scale $X$ and the long length scale $\tau$ defined by

$$
\omega(x)=\frac{2^{1 / 4}}{\left(x-z_{1}\right)^{1 / 4}} W(X, \tau), \quad \text { where } \quad X=\frac{\sqrt{V}\left(x-z_{1}\right)^{3 / 2}}{\sqrt{B}}, \quad \tau=\frac{3}{2} \log \left(x-z_{1}\right)
$$

Substituting (3..30) into (3..19), we find the transformed equation

$$
\begin{aligned}
W_{X X}+2 \sqrt{\frac{B}{V}}\left(x-z_{1}\right)^{-3 / 2} W_{\tau X}+\frac{B}{V} W_{\tau \tau}\left(x-z_{1}\right)^{-3}- & \frac{B}{V} W_{\tau}\left(x-z_{1}\right)^{-3} \\
& +\frac{5}{36} \frac{B}{V}\left(x-z_{1}\right)^{-2} W=\frac{2}{9}\left(\frac{1}{W^{3}}-W\right) .
\end{aligned}
$$

Expanding $W$ as

$$
W \sim W_{0}+\sqrt{\frac{B}{V}} \mathrm{e}^{-\tau} W_{1}+O(B)
$$


we find a succession of equations for $W_{0}, W_{1}, \cdots$, namely

$$
\begin{aligned}
W_{0 X X} & =\frac{2}{9}\left(\frac{1}{W_{0}^{3}}-W_{0}\right), \\
W_{1 X X}+\frac{2}{9}\left(1+\frac{3}{W_{0}^{4}}\right) W_{1} & =-2 W_{0 \tau X},
\end{aligned}
$$

and so forth.

Integrating the autonomous equation (3..33) once, we obtain

$$
W_{0 X}^{2}=\frac{2}{9}\left(A(\tau)^{2}+\frac{1}{A(\tau)^{2}}-W_{0}^{2}-\frac{1}{W_{0}^{2}}\right)
$$

where $A(\tau)$ is a function of the long length scale $\tau$ alone. The function $W_{0}(X, \tau)$ oscillates between the values $A(\tau)$ and $1 / A(\tau)$, so that $A(\tau)$ gives a measure of the amplitude of the oscillations. One more integration of equation (3..35) gives the general solution

$$
W_{0}(X, \tau)=\sqrt{A(\tau)^{2} \sin ^{2}\left[\frac{\sqrt{2}}{3} X-C(\tau)\right]+\frac{1}{A(\tau)^{2}} \cos ^{2}\left[\frac{\sqrt{2}}{3} X-C(\tau)\right]},
$$

where the arbitrary translation $C(\tau)$ likewise only depends on the long length scale $\tau$.

To find the amplitude $A$, we consider the solvability condition for the $O(\sqrt{B})$ governing equation (3..34), by insisting that $W_{1}(X, \tau)$ be a periodic function of $X$. It is evident that $W_{1}=W_{0 X}$ solves the homogeneous version of equation (3..34), and we therefore substitute $W_{1}=V W_{0 X}$ into (3..34) to find that $V$ satisfies the equation

$$
\frac{\mathrm{d}}{\mathrm{d} X}\left[W_{0 X}^{2} V_{X}\right]=-2 W_{0 X} W_{0 \tau X}
$$

Since $W_{0}$ and $W_{1}$ are periodic functions of the fast variable $X$, it follows that $V$ must be likewise. Hence, we deduce from (3..37) the solvability condition

$$
\int_{0}^{\lambda} W_{0 X} W_{0 \tau X} \mathrm{~d} X=0
$$

where $\lambda=3 \pi / \sqrt{2}$ is the wavelength of the periodic solution on the short length scale $X$. Integrating (3..38) by parts and making use of (3..33) we find

$$
\int_{0}^{\lambda} W_{0 X} W_{0 \tau X} \mathrm{~d} X=\left[W_{0 \tau} W_{0 X}\right]_{0}^{\lambda}+\frac{1}{9} \frac{\mathrm{d}}{\mathrm{d} \tau} \int_{0}^{\lambda}\left(\frac{1}{W_{0}^{2}}+W_{0}^{2}\right) \mathrm{d} X .
$$

We evaluate the final integral by using (3..35), and hence find

$$
\int_{0}^{\lambda} W_{0 X} W_{0 \tau X} \mathrm{~d} X=\frac{1}{2}\left[W_{0 \tau} W_{0 X}\right]_{0}^{\lambda}+\frac{\lambda}{9}\left(A(\tau)-\frac{1}{A(\tau)^{3}}\right) \frac{\mathrm{d} A(\tau)}{\mathrm{d} \tau} .
$$

The first term on the right-hand side of (3..40) is zero due to the periodicity of $W_{0}$, and we deduce that

$$
\frac{\mathrm{d} A}{\mathrm{~d} \tau}=0
$$


With $A$ equal to a constant, one integration of equation (3..37) leads to

$$
V_{X}=\frac{3}{\sqrt{2}} \frac{\mathrm{d} C}{\mathrm{~d} \tau},
$$

and from the periodicity of $V$ we infer that $\mathrm{d} C / \mathrm{d} \tau=0$. Therefore both $A$ and $C$ are constant even on the slow scale.

By substituting (3..30) into the initial conditions (3..19b) and letting $B \rightarrow 0$, we obtain the leadingorder conditions

$$
W_{0 X}=0, \quad W_{0}=\frac{\left(1-z_{1}\right)^{1 / 4} V^{1 / 4}}{2^{1 / 4}} \quad \text { at }(X, \tau)=\left(X^{*}, \tau^{*}\right),
$$

where

$$
X^{*}=\frac{\sqrt{V}\left(1-z_{1}\right)^{3 / 2}}{\sqrt{B}}, \quad \tau^{*}=\frac{3}{2} \log \left(1-z_{1}\right) .
$$

Applying the initial condition (3..43) to the leading-order solution (3..36), we evaluate the two integration constants:

$$
C=\frac{\sqrt{2}}{3} X^{*} \quad \text { (without loss of generality) }, \quad \text { and } \quad A=\frac{2^{1 / 4}}{V^{1 / 4}\left(1-z_{1}\right)^{1 / 4}} .
$$

Finally, reversing the scalings (3..30), we obtain the leading-order oscillatory solution

$$
\begin{aligned}
\omega(x) \sim\left(\frac{\left(1-z_{1}\right) V}{x-z_{1}}\right)^{1 / 4}\left\{\cos ^{2}\right. & {\left[\frac{\sqrt{2 V}}{3 \sqrt{B}}\left(\left(x-z_{1}\right)^{3 / 2}-\left(1-z_{1}\right)^{3 / 2}\right)\right] } \\
& \left.+\frac{2}{\left(1-z_{1}\right) V} \sin ^{2}\left[\frac{\sqrt{2 V}}{3 \sqrt{B}}\left(\left(x-z_{1}\right)^{3 / 2}-\left(1-z_{1}\right)^{3 / 2}\right)\right]\right\}^{1 / 2},
\end{aligned}
$$

in the region $z_{1}<x<1$. In Figure 11 we demonstrate that (3..46) has excellent agreement with the full numerical solution in $x>z_{1}$, except very close to $x=z_{1}$.

\section{4..3 Exponential solution: $-1<x<z_{1}$}

In the region $-1<x<z_{1}$, we anticipate that $\omega(x)$ is exponentially large, and therefore equation (3..19a) is approximated by

$$
\frac{B}{V} \omega^{\prime \prime} \sim-\frac{\left(x-z_{1}\right)}{2} \omega .
$$

Solving (3..47) in the limit $B \rightarrow 0$ we find the leading-order solution

$$
\omega \sim \frac{K}{\left(z_{1}-x\right)^{1 / 4}} \exp \left(\frac{\sqrt{2 V}}{3 \sqrt{B}}\left(z_{1}-x\right)^{3 / 2}\right),
$$

in the region $-1<x<z_{1}$, where $K$ is a constant.

In Figure 11, we compare the approximate solution (3..48) with a numerical solution of the full problem obtained following the procedure outlined in $\S 3.2$., with parameter values $B=10^{-3}, V=4$ and $\Theta_{\mathrm{MB}}=1$. With $K \approx 1.142$, the asymptotic and numerical solutions agree very well, except in a small neighbourhood of $x=z_{1}$. In general, the constant $K$ is determined as a function of the other parameters by matching across an inner layer at $x=z_{1}$, as described below. 


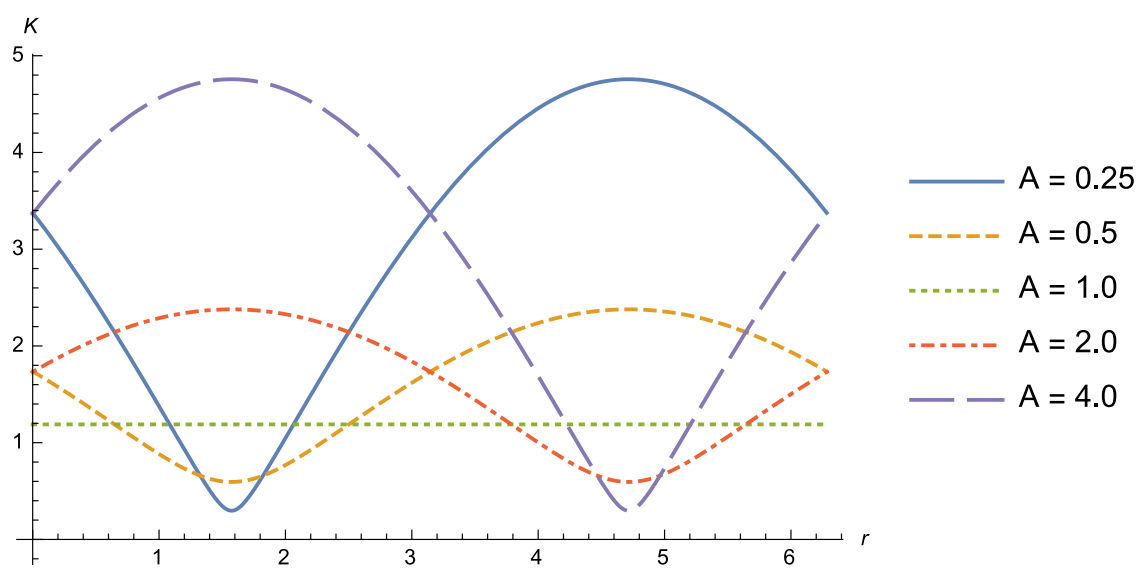

FIG. 12. The function $K(A, r)$ plotted against the remainder $r$ for various fixed values of $A$.

\section{4..4 Matching region: $x \approx z_{1}$}

We analyse the region $x \approx z_{1}$ by applying the scalings

$$
x=z_{1}+\left(\frac{B}{V}\right)^{1 / 3} \xi, \quad \omega(x)=\left(\frac{V}{B}\right)^{1 / 12} v(\xi),
$$

so that equation (3..19a) is transformed to the canonical form

$$
\frac{\mathrm{d}^{2} v}{\mathrm{~d} \xi^{2}}=\frac{1}{v^{3}}-\frac{\xi v}{2}
$$

Matching with the outer solutions (3..46) and (3..48) imposes the far-field behaviour

$$
\begin{array}{ll}
v(\xi) \sim\left(\frac{2}{\xi}\right)^{1 / 4}\left\{\frac{1}{A^{2}} \cos ^{2}\left[\frac{\sqrt{2}}{3} \xi^{3 / 2}-\frac{r}{2}\right]+A^{2} \sin ^{2}\left[\frac{\sqrt{2}}{3} \xi^{3 / 2}-\frac{r}{2}\right]\right\}^{1 / 2} & \text { as } \xi \rightarrow+\infty, \\
v(\xi) \sim \frac{K}{|\xi|^{1 / 4}} \exp \left(\frac{\sqrt{2}}{3}|\xi|^{3 / 2}\right) & \text { as } \xi \rightarrow-\infty,
\end{array}
$$

where the amplitude $A$ is given by equation (3..45), while $r \in[0,2 \pi)$ is the remainder such that

$$
\frac{2 \sqrt{2 V}}{3 \sqrt{B}}\left(1-z_{1}\right)^{3 / 2}=2 k \pi+r \quad \text { for } \quad k \in \mathbb{Z} \gg 1 .
$$

For given values of $A$ and $r$, the equation (3..50) and far-field condition (3..51a) in principle define $v(\xi)$ uniquely, and the final condition (3..52) thus determines $K(A, r)$ as a function of $A$ and $r$. In Figure 12, we use this procedure to plot $K(A, r)$ versus $r$ for various fixed values of $A$. For the special case $A=1$, the far-field condition (3..51a) is independent of $r$, and indeed we observe that $K(1, r)$ returns a constant value $\approx 1.18922$ independent of $r$. When $A \neq 1$, the far-field behaviour (3..51a) is invariant 
under the transformation $A \mapsto 1 / A, r \mapsto 2 \pi-r$, and we observe in Figure 12 that $K$ enjoys the symmetry $K(1 / A, 2 \pi-r) \equiv K(A, r)$.

With the particular parameter values $V=4, B=10^{-3}$ and $z_{1}=-1 / 2$, we find

$$
r=\frac{2 \sqrt{2 V}}{3 \sqrt{B}}\left(1-z_{1}\right)^{3 / 2}-34 \pi \approx 2.73036, \quad \text { and } \quad A=\left(\frac{2}{\left(1-z_{1}\right) V}\right)^{1 / 4}=\frac{1}{3^{1 / 4}},
$$

and by solving the problem $(3 . .50)-(3 . .51)$ we obtain $K\left(3^{-1 / 4}, 2.73036\right) \approx 1.142$. Figure 11 demonstrates the accuracy of the fit between asymptotic and numerical solutions when $K$ is found using the method outlined above.

\section{4..5 Total current density}

To evaluate the flux $J_{1}$, we substitute the asymptotic solution (3..48) into the boundary condition (3..20) to obtain

$$
J_{1} \sim-\frac{\sqrt{\left(1+z_{1}\right) V}}{K(A, r)^{2}} \exp \left(-\frac{2 \sqrt{2 V}}{3 \sqrt{B}}\left(1+z_{1}\right)^{3 / 2}\right)=-\frac{\sqrt{2 \Theta_{\mathrm{MB}}}}{K(A, r)^{2}} \exp \left(-\frac{8 \Theta_{\mathrm{MB}}^{3 / 2}}{3 V \sqrt{B}}\right),
$$

where

$$
A=\frac{1}{\left(V-\Theta_{\mathrm{MB}}\right)^{1 / 4}}, \quad r=\frac{8\left(V-\Theta_{\mathrm{MB}}\right)^{3 / 2}}{3 V \sqrt{B}}(\bmod 2 \pi),
$$

when we reintroduce the physical variable $\Theta_{\mathrm{MB}}$. Meanwhile, $J_{2}$ is still given by the asymptotic expression (3..29) found in $\S 3.3$. It follows that $J_{2} /\left|J_{1}\right|$ is exponentially small as $B \rightarrow 0$ with $V>\Theta_{\mathrm{MB}}$, and the total electron flux is therefore again given by $J \sim J_{1}$ to leading order, with $J_{1}$ now given by equation (3..54).

In Figure 13 we demonstrate the accuracy of the approximation (3..54) by comparing to the values of the current density obtained by numerical solution following the recipe described in $\S 3.2$., with parameter values $B=10^{-3}$ and $\Theta_{\mathrm{MB}}=1$. The asymptotic solution is remarkably successful in capturing the dramatic behaviour of $J$ as a function of $V$. The final exponential term in equation (3..54) causes $J$ to vary over many orders of magnitude as $V$ increases by a factor of 4 . The superimposed oscillatory dependence of the current density on the voltage arises from the prefactor containing the function $K(A, r)$ in equation (3..54). As shown in Figure 12, $K(A, r)$ is an oscillatory function of $r$ whose amplitude depends on $A$. The oscillations disappear when $A=1$, corresponding to $V=\Theta_{\mathrm{MB}}+1$, and indeed Figure 13 shows that the oscillatory amplitude becomes very small close to $V=2$ when $\Theta_{\mathrm{MB}}=1$.

\section{Discussion and conclusions}

In this paper, we have investigated two different approaches to introduce quantum mechanical effects into continuum models for charge transport. DGC theory involves the addition of a higher order quantum term into the drift-diffusion equations. The physically relevant limit where this term is small results in a singular perturbation problem, which is naturally analysed using boundary layer theory. We illustrated such an approach while using DGC theory to incorporate quantum mechanical effects into the DD model proposed in Black et al. (2013) for electron flow across a thin glass layer between two electrodes. We 


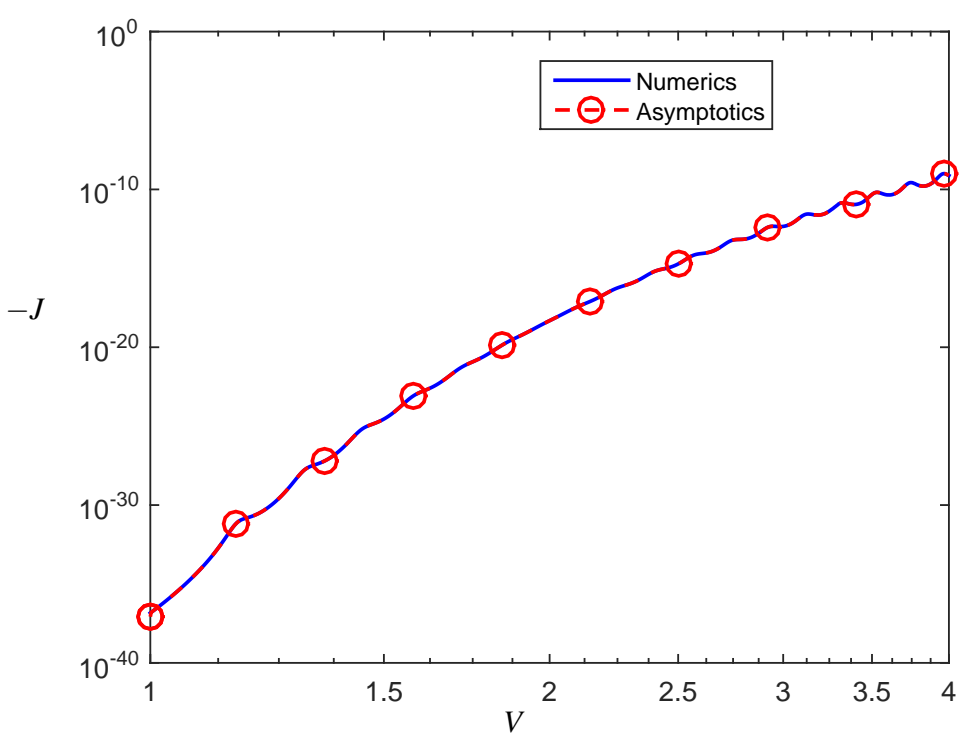

FIG. 13. Current density $(-J)$ plotted against applied potential difference $V$ with parameter values $B=10^{-3}$ and $\Theta_{\mathrm{MB}}=1$. The asymptotic solution [red circles] is given by (3..54) and the numerical solution [blue solid] is found by solving the system $(3 . .19)-(3 . .20)$.

considered the physically-relevant limit where the Debye length and the electron quantum length are similar in size and both much smaller than a typical device lengthscale. We found that the introduction of the quantum term may profoundly change the behaviour of the electron density profile in boundary layers at the edges of the domain, but leaves the leading-order outer problem unchanged in all parameter regimes explored. The net contact resistance of the layer is therefore not significantly altered by the inclusion of quantum effects, implying that they would be very difficult to detect experimentally.

The most interesting new phenomenon to occur due to the addition of quantum effects occurs in the "large current" regime, where the electron flux plays an important role in the downstream boundary layer. Here, we find that the quantum term causes oscillatory variations in the distribution of the electron density which are not predicted by DD theory. We are unaware of the oscillatory behaviour being reported in other studies that make use of the DGC theory.

We also investigated the alternative DGT theory by extending the work of Ancona (1990). Our reformulation of the boundary value problem into an initial value problem enables easy numerical solution and allows us to observe previously unreported oscillatory behaviour in the solution profile of the electron density and the current density. The numerical solution was shown to have excellent agreement with asymptotic calculations. Furthermore, through asymptotic analysis we were able to fully characterise the observed oscillatory behaviour and obtain accurate analytic expressions for the total current density which are comparable to those from more complicated microscopic quantum transport theories (see Simmons (1963)).

As noted above, our asymptotic analysis and careful numerical calculations revealed oscillatory behaviour in the solution structure of the electron density predicted by both theories, at least in some parameter regimes. We are unaware of the oscillatory behaviour being reported in any previous studies that make use of either DGC or DGT theory. However, oscillatory behaviour of the electron density 
is often found when using the nonequilibrium Green's function method to model quantum mechanical tunnelling and is attributed to quantum mechanical electric charge screening (Friedel oscillations) (see Janta-Polczynski et al. (2008); Ancona (2011)).

\section{Funding}

This work was supported by EPSRC and DuPont (UK) Ltd through mathematics CASE award number $\mathrm{BK} / 10 / 040$. In compliance with EPSRC's open access initiative, the data in this paper is available from http://dx.doi.org/10.5287/bodleian:kKvV9BMzK.

\section{References}

Abebe, H., \& Cumberbatch, E. (2003) Quantum mechanical effects correction models for inversion charge and current-voltage (IV) characteristics of the MOSFET device, In Proceedings 2003 nanotechnology Conference, 218-221.

Ancona, M.G.(1990) Macroscopic description of quantum-mechanical tunneling, Physical Review $B, \mathbf{4 2}(2), 1222$.

Ancona, M.G.(1992) Density-gradient analysis of field emission from metals, Physical Review B, 46(8), 4874

ANCONA, M.G.(2011) Density-gradient theory: a macroscopic approach to quantum confinement and tunneling in semiconductor devices, Journal of Computational Electronics, 10(1-2), 65.

AnCONA, M.G. \& IAFrATE, G.J.(1989) Quantum correction to the equation of state of an electron gas in a semiconductor, Physical review B, 39(13), 9536.

Ancona, M.G. \& Svizhenko, A.(2008) Density-gradient theory of tunneling: Physics and verification in one dimension, Journal of Applied Physics, 104(07), 073726.

Ancona, M.G. \& Tiersten, H.F.(1987) Macroscopic physics of the silicon inversion layer, Physical Review B, 35(15), 7959-7965.

Ancona, M.G., Yergeau, D., Yu, Z. \& Biegel, B.A.(2002) On Ohmic boundary conditions for density-gradient theory, Journal of Computational Electronics, 1(1-2), 103-107.

Ancona, M.G., Yu, Z., Lee, W.C., Dutton, R.W., Voorde, P.J., CAO, M. \& Vook, D.(2000) Density-gradient analysis of MOS tunneling, IEEE transactions on Electron Devices, 47(12), 23102319.

Ancona, M.G., Yu, Z., Lee, W.C., Dutton, R.W. \& Voorde, P.V.(1997) Density-gradient simulations of quantum effects in ultra-thin oxide MOS structures, In SISPAD, 97-100.

Asenov, A., Slavcheva, G., Brown, A.R., Davies, J.H. \& Saini, S.(2001) Increase in the random dopant induced threshold fluctuations and lowering in sub-100 nm MOSFETs due to quantum effects: a 3-D density-gradient simulation study. IEEE Transactions on Electron devices, 48(4), 722729.

BLACK, J.P. (2015) Mathematical modelling of electronic contact mechanisms in silicon photovoltaic cells. Thesis (PhD). University of Oxford. 
Black, J.P., BREWARD, C.J.W., Howell, P.D., Young, R.J.S.(2013) Mathematical modeling of contact resistance in silicon photovoltaic cells, SIAM Journal on Applied Mathematics, 73(5), 19061925.

Cumberbatch, E., Uno, S. \& Abebe, H. (2006) Nano-scale MOSFET device modelling with quantum mechanical effects, European Journal of Applied Mathematics, 17(4), 465-489.

Damle, P.S., Ghosh, A.W. \& DatTA, S.(2002) First-principles analysis of molecular conduction using quantum chemistry software, Chemical Physics, 281(2), 171-187.

Damle, P.S., Ghosh, A.W. \& DatTA, S.(2001) Unified description of molecular conduction: From molecules to metallic wires, Physical Review B, 64(20), 201403.

Janta-Polczynski, B.A., Cerdá, J.I., Éthier-Majcher, G., Piyakis, K. \& Rochefort, A.(2008) Parallel scanning tunneling microscopy imaging of low dimensional nanostructures, Journal of Applied Physics, 104(2), 023702.

Markowich, P.A.(1986) The Stationary Semiconductor Device Equations. New York: SpringerVerlag.

PINNAU, R.(1999) A note on boundary conditions for quantum hydrodynamic equations, Applied mathematics letters, 12(5), 77-82.

Pinnau, R.(2002) A review on the quantum drift diffusion model, Transport Theory and Statistical Physics, 31(4-6), 367-395.

Sano, N, Hiroki, A. \& Matsuzawa, K.(2002) Device modeling and simulations toward sub-10nm semiconductor devices, IEEE Transactions on Nanotechnology,1(1), 63-71.

Simmons, J.G.(1963) Generalized formula for the electric tunnel effect betwen similar electrodes separated by a thin insulating film, Journal of Applied Physics, 34(6), 1793.

SzE, S.M. \& NG, K.K.(2006) Physics of semiconductor devices. New Jersey: John Wiley \& Sons.

Uno, S., Abebe, H., Cumberbatch, E.(2007) Analytical description of inversion-layer quantum effects using the density gradient model and singular perturbation theory, Japanese Journal of Applied Physics, 4612R, 7648 .

Verwey, J.F., Amerasekera, E.A. \& Bisschop, J.(1990) The Physics of $\mathrm{SiO}_{2}$ layers, Reports on Progress in Phyics, 53(10), 1297-1331.

Watling, J.R., Brown, A.R. \& Asenov, A.(2002) Can the density gradient approach describe the source-drain tunneling in decanano double-gate MOSFETs? Journal of Computational Electronics, 1(1), 289-293. 